\title{
Power Gain Optimization for Multiple Active Multiple Passive Dipole Antenna Arrays
}

by

\author{
Shady Elkamhawy
}

A thesis submitted to the Faculty of Graduate and Postdoctoral Affairs in partial fulfillment of the requirements for the degree of

\section{Master of Applied Science}

in

Electrical and Computer Engineering

Carleton University

Ottawa, Ontario

(C) 2020

Shady Elkamhawy 


\begin{abstract}
In this work we consider dipole antenna arrays composed of both active, i.e., excited by voltage sources, and reactively controlled passive dipole elements. Unlike the traditional approach in which the current distribution along the antenna elements is assumed to be sinusoidal, herein we obtain the exact distribution from the Hallen equations using the Method of Moments (MoM). The obtained current distributions are subsequently used to derive exact expressions for the far-field power gain of the antenna array in any given direction. Using the exact current distribution, we show that the current distributions on the dipole elements can deviate significantly from the sinusoidal approximation. Consequently, the exact power gain pattern mismatches that obtained using the sinusoidal assumption for the current distribution. Optimizing the excitation voltages and the load reactances for the active and passive elements, respectively, to maximize the gain; constitutes a non-convex optimization problem. To circumvent this difficulty, we develop an efficient algorithm that yields beam patterns close to those that would be yielded by the all-active analog of the considered antenna array. The advantage of our proposed approach over the one based on sinusoidal approximation of current distributions is demonstrated by numerical evaluation of the derived analytical expressions and verified using the Numerical Electromagnetic Code (NEC) simulator.
\end{abstract}


To my family, friends and professors 


\section{Contents}

Page

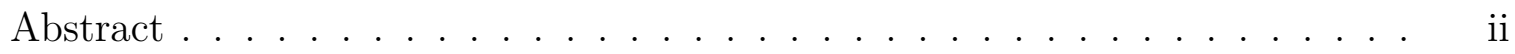

Dedication ........................ iii

List of Figures $\ldots \ldots \ldots \ldots \ldots \ldots \ldots \ldots \ldots \ldots \ldots$ vii Chapters:

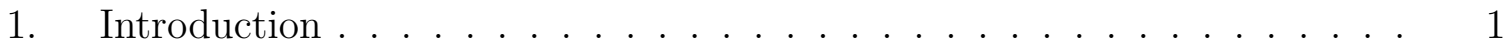

1.1 Motivation . . . . . . . . . . . . . . . . 1

1.2 Related Work . . . . . . . . . . . . . . . . . . . 3

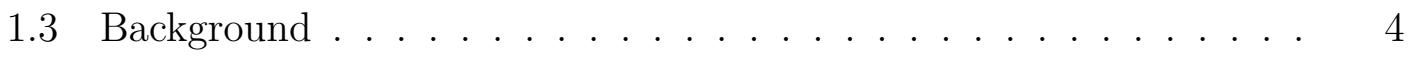

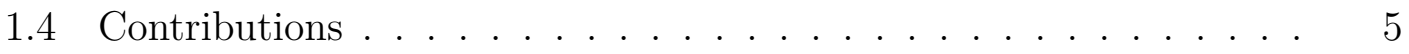

1.5 Notation . . . . . . . . . . . . . . . . . 6

2. Formulation of the Multiple-Active Multiple-Passive Dipole Antenna Array 7

2.1 System Model . . . . . . . . . . . . . . . . . . . . . . . 7

2.1 .1 Array Configuration . . . . . . . . . . . . . . 8

2.1 .2 Steering Vector . . . . . . . . . . . . . . . . 8

2.1 .3 Terminating Circuit . . . . . . . . . . . . . 9

2.1.4 Power Gain of Radiation Pattern . . . . . . . . . . . . 10

2.2 Computation of the Current Distribution . . . . . . . . . . . 11

2.2.1 Hallen Equations for Coupled Dipoles . . . . . . . . . . 12

2.2 .2 Exact Current Distribution . . . . . . . . . . . . . . . 13

2.2.3 The Sinusoidal Approximation of the Current Distribution . 15

2.2.4 Comparison Between The Exact and Approximate Current Distributions . . . . . . . . . . . . . . 16

2.3 Calculation of the Power Gain . . . . . . . . . . . . . 17 
2.3.1 The Power Gain as a Rayleigh Quotient: Exact . . . . . . . 17

2.3.2 The Power Gain as a Rayleigh Quotient: Approximate . . . 17

2.3.3 The Power Gain as a Weighted Pattern: Exact . . . . . . . 18

2.3.4 The Power Gain as a Weighted Pattern: Approximate . . . 18

2.3.5 Comparison Between the Power Gain of the Exact and Sinusoidal Approximation Current Distributions . . . . . . . . . 19

2.4 Power Gain Optimization . . . . . . . . . . . . . . . . . . . 19

2.4.1 An Efficient Algorithm for Optimizing the Power Gain of Antenna Arrays With Active and Passive Elements . . . . . 21

3. Numerical Results . . . . . . . . . . . . . . . . . . . . . . . . 26

3.1 Error in Sinusoidal Current Distribution for an Array of two Elements 27

3.2 Error in the Power Gain Pattern of the Harrington Array . . . . . 29

3.3 Optimization of the Harrington Array . . . . . . . . . . . . . . . 31

3.4 Optimization of a circular array with 3 active and 9 passive elements 32

4. Conclusion and Future Work . . . . . . . . . . . . . . . . 37

4.1 Conclusion . . . . . . . . . . . . . . . . . . . 37

4.2 Future Work . . . . . . . . . . . . . . . . . . . . . . . 38

Appendices:

A. The Method of Moments $(\mathrm{MoM}) \ldots \ldots$. . . . . . . . . . . 39

A.1 Basis Function Expansion . . . . . . . . . . . . . . . . . 39

A.1.1 Mutual and Self Impedance Matrices . . . . . . . . . . . . . 40

A.1.2 End of Wire Condition . . . . . . . . . . . . . . . . . 41

A.1.3 Solving for the Current Distribution Vector . . . . . . . . . 41

B. Sinusoidal Approximation of The Current Distribution . . . . . . . . . . 43

B.1 Kernel Approximation as a Scaled Delta Function . . . . . . . . . . 43

B.1.1 End of Wire Condition . . . . . . . . . . . . . . . . 44

B.1.2 Sinusoidal Distribution . . . . . . . . . . . . . . . . 44

B.1.3 Matrix Form ................ . . 45

C. Power Gain as a Rayleigh Quotient . . . . . . . . . . . . . . . . . . 47 
D. Representation of Excitation Voltage vector in Angular Coordinates and Phase shifts . . . . . . . . . . . . . . . . 48

E. Gradient of the Power Gain with respect to load reactances _ . . . . . 49

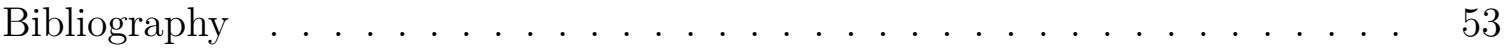




\section{List of Figures}

Figure

Page

$2.12 \mathrm{D}$ array of dipoles . . . . . . . . . . . . . . . . . . 8

2.2 Illustration of the phase shift in the azimuth plane . . . . . . . . 9

2.3 Terminating circuit for the $n$-th dipole . . . . . . . . . . . 10

2.4 Flowchart of the optimization algorithm in Section 2.4. . . . . . . . . 25

3.1 A Harrington array with $N=7$ dipoles. . . . . . . . . . . . . 27

3.2 Uniform circular array with 3 active and 9 passive elements. . . . . . 28

3.3 Current error for an array composed of 1 active and 1 passive, for load reactance $X \ldots \ldots \ldots \ldots \ldots$

3.4 Polar plot in the azimuth plane of the expected and actual power gains for beam direction at $\phi_{0}=0^{\circ}, 10^{\circ}, 20^{\circ}, 30^{\circ}$ using the reactances obtained in $[1] \ldots \ldots \ldots \ldots \ldots \ldots \ldots . \ldots \ldots$

3.5 Polar plot in the azimuth plane of the expected and actual power gains of the Harrington array, for beam direction at $\phi_{0}=0^{\circ}, 10^{\circ}, 20^{\circ}, 30^{\circ}$ using the reactances obtained from the proposed optimization algorithm. 33

3.6 Polar plot in the azimuth plane of the expected and actual power gains of the 3 active and 9 passive circular array, for beam direction at $\phi_{0}=0^{\circ}, 20^{\circ}, 40^{\circ}, 60^{\circ}$ using the reactances obtained from the proposed optimization algorithm. . . . . . . . . . . . . . 


\section{LIST OF ABBREVIATIONS}

\begin{tabular}{ll}
\hline Symbol & Definition \\
\hline$\overline{\text { BS }}$ & Base Station \\
UE & User Equipment \\
RF & Radio Frequency \\
DC & Direct Current \\
MoM & Method of Moments \\
NEC & Numerical Electromagnetic Code \\
\hline
\end{tabular}




\section{LIST OF SYMBOLS}

\begin{tabular}{ll}
\hline Symbol & Definition \\
\hline$\overline{\mathcal{N}_{a}}$ & Set of indices of active elements \\
$\mathcal{N}_{p}$ & Set of indices of passive elements \\
$N$ & Number of elements in the array \\
$\lambda$ & Wavelength of the waveforms \\
$k$ & Wavenumber of the waveform \\
$\phi$ & azimuth angle \\
$\theta$ & elevation angle \\
$\boldsymbol{a}$ & Steering vector \\
$\boldsymbol{I}$ & Current distribution vector \\
$\boldsymbol{V}$ & Excitation voltages vector \\
$\boldsymbol{X}$ & Load Reactances vector \\
$P$ & Input power \\
$G_{a}$ & Azimuth power gain \\
$\eta$ & Intrinsic impedance of free space \\
$h$ & half-length of the dipole \\
$r$ & radius of the dipole \\
$Q$ & Number of samples per half-dipole \\
$\mathbf{I}$ & Identity matrix \\
\hline
\end{tabular}




\section{Chapter 1}

\section{Introduction}

\subsection{Motivation}

Future wireless communication systems will capitalize on massive Multiple-Input Multiple-Output (MIMO) techniques to achieve high gains in throughput and reliability [2], which also dictate the utilization of higher frequency bands to support larger bandwidths. Increasing the frequency leads to an increase in the path-loss. Luckily, in the aforementioned systems, Base Stations (BSs) are equipped with a large number of antennas that will use beamforming to communicate to User Equipment devices (UEs), which are expected to have a small number of antennas each [3]. Beamforming enables the emitted and received powers to be focused in the direction of desired users. This directly translates to significant power savings and an innate ability to avoid interference from other UEs in the system [4].

Beamforming is realized by using antenna arrays with particular configurations, i.e., geometry, spacing, and number of elements, in addition to the shape of individual antennas. The desired beam pattern is formed by controlling the current that flows through each element, which subsequently controls the contribution of the antenna

element to the radiated field [2]. An antenna array can have both, active elements, 
i.e., excited by a voltage or a current source, or passive. In the latter case, the antenna is terminated by a load impedance. In the case of active antennas, the beam pattern and the direction of maximum radiation are controlled by the amplitude and phase of the excitation voltages or currents of individual elements, whereas in the case of passive antennas, these features are determined by the loading impedances terminating the antenna elements. In particular, varying these impedances controls the current induced due to coupling effects with other array elements [1], and hence, controls the radiation pattern.

In comparison with their passive counterparts, all-active antenna arrays yield more directive beam patterns and subsequently higher power gains [2]. However, as the number of active elements increases, the feed network to the antenna array becomes more complex. This is not only because of the cost of the radio frequency (RF) chains needed to excite the antenna elements, but also because of the elaborate synchronization required to control the relative phases between excitation signals. These difficulties are significantly alleviated by replacing some of the active elements with passive ones, which are characterized by much simpler feed networks; as they require neither RF chains nor synchronization thereof [5]. The terminating load of the passive elements is typically chosen to be purely reactive, thereby does not consume active power and can be readily implemented using cost-effective varactor diodes controlled by DC voltages [5]. These advantages of passive antenna arrays render them attractive for practical implementation of beamforming in wireless communication systems. 


\subsection{Related Work}

Due to its central role in improving coverage and reliability in the upcoming 5G architecture, beamforming optimization and design have received renewed interest [6], [7], [8], and although all-active antenna arrays have been considered, the advantages of their passive counterparts render them more amenable to meet the stringent spatial and energy constraints imposed by the $5 \mathrm{G}$ architecture. In fact, the deployment of antenna arrays with both active and passive elements has been considered not only for UEs [3], where device compactness and energy efficiency are of paramount importance, but also for base stations, where beam steering capabilities comparable to those of all-active arrays were shown to be achievable with significantly lower deployment cost and complexity [4]. Antenna arrays can have either one or multiple active elements in addition to one or more passive elements. For instance, in [1], one active element is surrounded by multiple uniformly-spaced reactively loaded passive elements. In contrast, antenna configurations with multiple active and multiple passive elements have been considered in [2]. It was shown therein that a performance comparable to that of purely active antenna arrays can be achieved, but with only half the number of elements being active. Alternatively, simpler antenna arrays could also be implemented with switched loads instead of reactive loads [9], which restricts the antenna array to switch between a finite set of directive radiation patterns. Popular designs of antenna arrays typically involve dipoles or monopoles due to their simple modelling, rendering calculations significantly simpler.

Despite the promises offered by antenna arrays with passive elements, using them for effective beamforming comes at a high computational cost. In particular, whereas 
the determination of the feed currents and/or voltages in the case of all-active antenna arrays is relatively straightforward [10], the computations required to optimize the reactive loads for the passive elements is rather involved. Several approaches have been considered in the literature, some based on analytical techniques [11] and others based on numerical ones $[1,5]$. An implicit assumption in these approaches is that the current distribution on the elements of the passive antennas has a sinusoidal form. Despite the popularity of these approaches, the actual current distribution is known to be not necessarily sinusoidal and depends primarily on the loading reactances, excitation voltages and antenna geometry. Hence, this distribution may deviate significantly from any predetermined form [12]. Notwithstanding this discrepancy, the sinusoidal assumption is prevalent in the overwhelming majority of analysis pertaining to passive antenna arrays, irrespective of its potentially significant impact on the calculation of the resulting radiation pattern, see e.g., $[1,5,11,13]$.

\subsection{Background}

One approach for finding the exact current distribution for general loading impedance values is to solve the Hallen integro-differential equations for coupled dipoles [14]. This process is usually computationally demanding, which might be the key reason for the popularity of the sinusoidal approximation. Solving the Hallen equations involves the use of the Method of Moments (MoM), whereby the continuous current distribution on each dipole is discretized and the resulting system of linear equations is solved, for given feed voltages and load impedances. In a complementary fashion, for a given current distribution, the reactive load impedance required to steer the beam in a particular direction could be obtained by invoking intricate multivariate 
search techniques [1]. The multivariate search can be simplified by using sequential searches, rather than joint ones, see e.g., [1]. In fact, one of our contributions in this work is to provide a simplified form of the exact current distribution. In particular, in this form the effect of the array geometry is separated from the effect of the load impedances, which greatly simplifies the evaluation of effective impedances, exact current distributions, and the power gain of the antenna array.

\subsection{Contributions}

Our goal in this thesis is to optimize the excitation voltages and load impedances of passive antenna arrays while taking into consideration the actual current distributions, rather than simplified versions thereof. Towards that end, we will use the Hallen integro-differential equations to obtain explicit expressions of the gain of the antenna array as a function of excitation voltages and load impedances. Unfortunately, this expression is rather cumbersome and results in a non-convex optimization problem. To solve this problem, we develop a novel technique that hinges on the observation that the gain achieved by a passive antenna array is upper-bounded by the gain achieved by an all-active antenna array, with the same geometry and configuration. This bound serves not only to guide the search for the final impedances, but also to provide performance assurances.

Numerical evaluation of the derived analytical expressions, supported by the results obtained from the Numerical Electromagnetic Code (NEC) simulator, suggests that the antenna patterns corresponding to the actual current distributions can differ significantly from those corresponding to the commonly-assumed sinusoidal ones. 
Finally, we show that our methodology yields a substantial advantage over the one proposed in [1].

\subsection{Notation}

We will use standard notation throughout. In particular, the transpose and the Hermitian transpose operators will be denoted by $(\cdot)^{\top}$ and $(\cdot)^{\dagger}$, respectively. The identity matrix of size $N$ will be denoted by $\mathbf{I}_{N}$. The $\operatorname{diag}\{\cdot\}$ denotes the diagonal matrix in which the entries of the principal diagonal are those of the vector argument. The real part of a complex number will be denoted by $\Re\{\cdot\}$. 


\section{Chapter 2}

\section{Formulation of the Multiple-Active Multiple-Passive Dipole Antenna Array}

In this chapter, we provide the mathematical framework for a generic dipole antenna array comprising an arbitrary number of active and passive elements. In particular, we will provide a mathematical representation of the array configuration, the steering vector, the terminating circuit of each dipole, and the power gain of array as a function of the current distributions on the dipoles and the total power input to the array. Then, we provide the methodology by which the exact current distribution is obtained and compare it with the sinusoidal approximation. Consequently, we simplify the power gain expressions in terms of the intrinsic and extrinsic parameters of the antenna array. Then, an optimization algorithm is presented to maximize the gain for a given direction.

\subsection{System Model}

In this section, we provide the mathematical definitions for the extrinsic and intrinsic parameters of antenna array. 


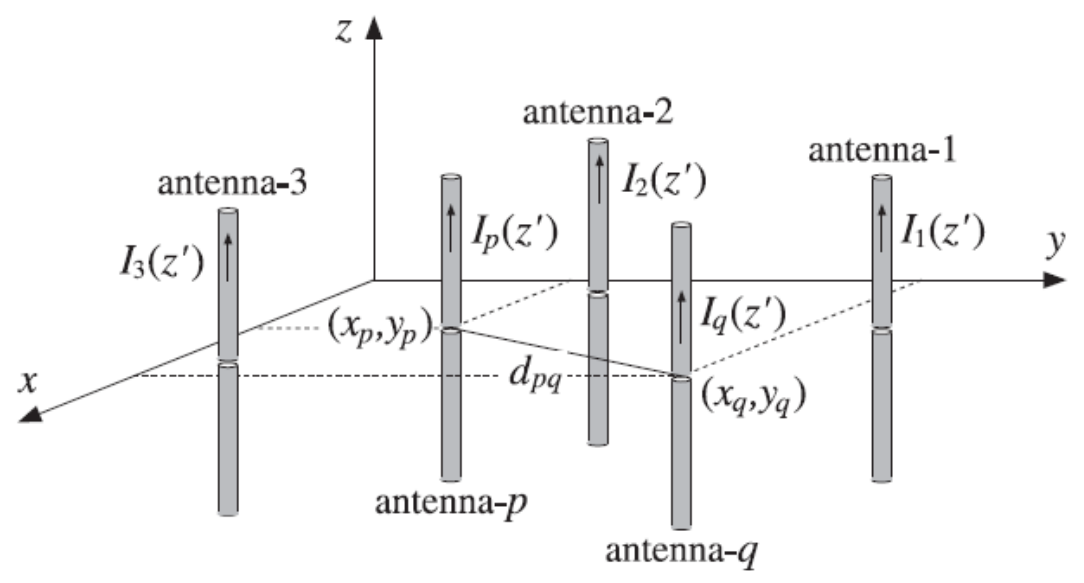

Figure 2.1: 2D array of dipoles

\subsubsection{Array Configuration}

In Fig. 2.1, we consider an array of $N$ identical center-fed dipoles of radius $r$ and length $2 h$. The dipoles are parallel to the $z$-axis and their centers lie in the $x y$-plane at $\left\{x_{n}, y_{n}\right\}_{n=0}^{N-1}$. Let $\mathcal{N}_{a}$ and $\mathcal{N}_{p}$ denote the sets containing the indices of the active and passive elements, respectively.

\subsubsection{Steering Vector}

The locations, $\left\{x_{n}, y_{n}\right\}_{n=0}^{N-1}$, of the antenna elements result in a relative phase shift in the response of each dipole. This is captured in the steering vector as shown below. In particular, using $k=\frac{2 \pi}{\lambda}$ to denote the wave-number corresponding to the wavelength $\lambda$ of the sinusoidal excitation source, it can be readily verified that at angle $\phi$ in the azimuth plane, i.e., at an elevation angle $\theta=\frac{\pi}{2}$ (as shown in Fig. 2.2), the phase of the wavefront at the $n$-th element lags that at the origin by [14]

$$
\rho_{n}(\phi)=k_{x}(\phi) x_{n}+k_{y}(\phi) y_{n},
$$

where $k_{x}(\phi)=k \cos \phi$ and $k_{y}(\phi)=k \sin \phi$. 


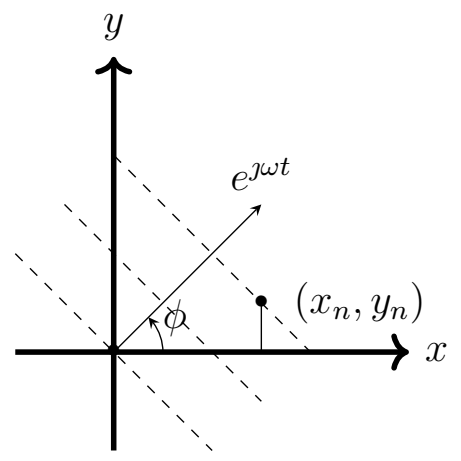

Figure 2.2: Illustration of the phase shift in the azimuth plane

Using (2.1), the steering vector of the array can be expressed as [14]

$$
\boldsymbol{a}(\phi)=\left[a_{0}(\phi) \cdots a_{N-1}(\phi)\right]^{\top}
$$

where $a_{n}(\phi)=e^{-\jmath \rho_{n}(\phi)}, n=0, \ldots, N-1$.

\subsubsection{Terminating Circuit}

The terminating circuit of the $n$-th element can be modelled using an excitation sinusoidal voltage source $V_{n}$ in series with a load reactance $X_{n}$ [1], as shown in Fig. 2.3. (Note that to avoid power wastage, load resistances are not considered in the terminating circuit.) The current distribution along the dipole is denoted by $I_{n}(z)$, with $I_{n}(0)$ denoting the feed-current.

From Fig. 2.3, it can be seen that, for any dipole element, being active or passive, the voltage between the terminals of the dipole is given by

$$
V_{f_{n}}=V_{n}-\jmath X_{n} I_{n}(0)
$$

We note that both the load reactance of an active element and the excitation voltage of a passive element are assumed to be zero. For notational convenience, the feed and excitation voltages and load reactances for all antenna elements will be 


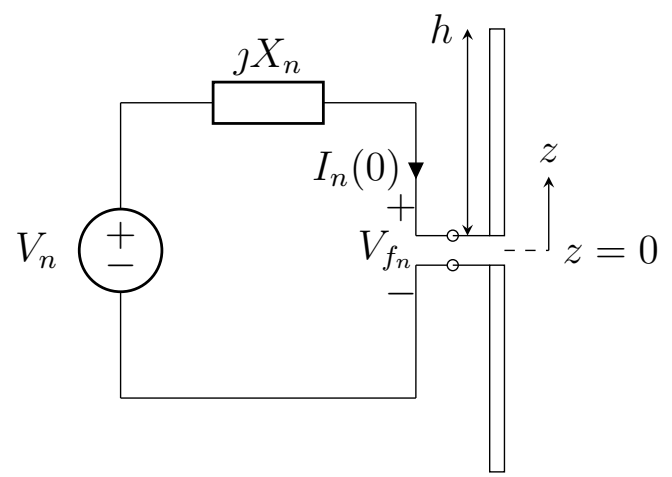

Figure 2.3: Terminating circuit for the $n$-th dipole

expressed in the following vector form:

$$
\begin{gathered}
\boldsymbol{V}_{f}=\left[\begin{array}{lll}
V_{f_{0}} & \cdots & V_{f_{N-1}}
\end{array}\right]^{\top}, \\
\boldsymbol{V}=\left[\begin{array}{lll}
V_{0} & \cdots & V_{N-1}
\end{array}\right]^{\top}, \\
\boldsymbol{X}=\left[\begin{array}{lll}
X_{0} & \cdots & X_{N-1}
\end{array}\right]^{\top} .
\end{gathered}
$$

Similarly, the current distribution vector at any point $z$ along the $z$-axis is denoted by

$$
\boldsymbol{I}(z)=\left[I_{0}(z) \cdots I_{N-1}(z)\right]^{\top}
$$

Using this notation, the total input power to the antenna array can be expressed as $[14]$

$$
P=\frac{1}{2} \Re\left\{\boldsymbol{I}^{\dagger}(0) \boldsymbol{V}\right\}
$$

\subsubsection{Power Gain of Radiation Pattern}

The amount of power delivered to a distant receiver located at angle $\phi$ in the azimuth plane, i.e. an elevation angle $\theta=\frac{\pi}{2}$, is proportional to the far-field array gain, which, for a given array geometry, depends on the load reactance vector, $\boldsymbol{X}$, 
and the excitation voltage vector, $\boldsymbol{V}$. In particular, we can write [14]

$$
G_{a}(\phi \mid \boldsymbol{V}, \boldsymbol{X})=\frac{\eta k^{2}}{8 \pi P}\left|\int_{-h}^{h} \boldsymbol{a}^{\dagger}(\phi) \boldsymbol{I}(z) d z\right|^{2}
$$

where $\eta \approx 376.73 \Omega$ is the intrinsic impedance of free space and $P$ is the total input power defined in (2.8). The power gain for a given azimuth direction $\phi$ represents the amount of power delivered to a receiver in that direction if the input power is $1 W$. In other words, it is the normalized radiation intensity in a given direction with respect to an equivalent isotropic radiator.

From (2.9), it can be seen that the array gain depends explicitly on the current distribution on the elements of the array. Hence, this distribution ought to be calculated accurately.

Deviating from the traditional approach of assuming fixed sinusoidal current distributions along the dipoles $[1,2,5]$, the actual current distributions on the dipoles can be obtained numerically by solving the Hallen equations for coupled dipoles [14] for a given array geometry, a set of load impedances, and excitation voltages.

\subsection{Computation of the Current Distribution}

To calculate the power gain of the antenna array at a given azimuth angle, $\phi$, the current distribution on each element must be computed. This can be achieved by solving the Hallen equations for coupled dipoles using the MoM numerical technique. In this section we present the current distribution resulting from solving the Hallen equations with MoM technique. For comparison, we present the sinusoidal approximation and provide an expression of the error resulting from this approximation. 


\subsubsection{Hallen Equations for Coupled Dipoles}

The Hallen equations for coupled dipoles are obtained by invoking the boundary conditions for the electric field at the surface of each dipole [14]. More precisely, the dipoles are modelled as perfect conductors which imply that the total tangential component (z-component) of the electric field at the surface of each dipole must be equal to zero. The tangential component of the electric field is composed of the superposition of: 1- the electric field due to the voltage between the terminals of the dipole, and 2- the electric field induced by the dipoles in the array. Writing this condition explicitly yields the following lemma, see e.g., [14]

Lemma 1. Consider a set of $N$ identical thin-wire centre-fed parallel dipoles, each with radius $r$ and length $2 h$ excited with the feed voltage vector $\boldsymbol{V}_{f}$ in (2.4). The current distribution, $I_{n}$ along the $n$-th dipole must satisfy the following equation:

$\frac{j \eta}{2 \pi} \sum_{n=0}^{N-1} \int_{-h}^{h} I_{n}\left(z^{\prime}\right) K\left(z-z^{\prime}, d_{m, n}\right) d z^{\prime}=C_{m} \cos (k z)+V_{f_{m}} \sin (k|z|), m=0, \ldots, N-1$,

where

$$
\begin{aligned}
& K\left(z-z^{\prime}, d_{m, n}\right)=\frac{1}{2 \pi} \int_{0}^{2 \pi} \frac{e^{-\jmath k S_{m, n}}}{S_{m, n}} d \phi^{\prime}, \\
& S_{m, n}=\sqrt{\left(z-z^{\prime}\right)^{2}+d_{m, n}^{2}+r^{2}-2 r d_{m, n} \cos \left(\phi^{\prime}\right),} \\
& d_{m, n}= \begin{cases}\sqrt{\left(x_{m}-x_{n}\right)^{2}+\left(y_{m}-y_{n}\right)^{2}}, & m \neq n, \\
r, & m=n .\end{cases}
\end{aligned}
$$

The constants $\left\{C_{m}\right\}_{m=0}^{N-1}$ in (2.10) are chosen such that $I_{m}(h)=I_{m}(-h)=0, m=$ $0, \ldots, N-1$.

The function $K\left(z-z^{\prime}, d_{m, n}\right)$ in $(2.11)$ is called thin-wire kernel of the dipole [14] and its evaluation can be performed efficiently using the techniques in [15] and [16]. 


\subsubsection{Exact Current Distribution}

From the Hallen equation in (2.10) and the definition of $\boldsymbol{V}_{f}$ in (2.4), it can be seen that, when the load reactances $\boldsymbol{X}$, the excitation voltages $\boldsymbol{V}$ and the geometry of the array are known, the current distributions on the dipoles can be obtained.

The complexity of (2.10) renders the derivation of analytical expressions of the current distributions intractable. A common approach to circumvent this difficulty is to obtain these distributions numerically using the MoM technique. This technique relies on taking $(2 Q+1)$ samples of the current distribution along each dipole and solving the set of resulting linear equations corresponding to (2.10). This technique is summarized in Appendix A [14]. Applying the MoM to the Hallen equations yields the following result.

Lemma 2 (Exact Current Distribution). Given excitation voltages vector, $\boldsymbol{V}$, and load reactances vector, $\boldsymbol{X}$, the sampled current distribution vector $\boldsymbol{I}$ obtained from the MoM can be expressed as

$$
\boldsymbol{I}=\boldsymbol{M}\left(\mathbf{I}_{N}+\jmath \operatorname{diag}\{\boldsymbol{X}\} \boldsymbol{U}_{f} \boldsymbol{M}\right)^{-1} \boldsymbol{V}
$$

where $\boldsymbol{I}$ is the vector constructed by stacking the $N$ vectors $\left\{\boldsymbol{I}_{n}\right\}_{n=0}^{N-1}$ corresponding to the $N$ dipoles on top of each other, $\mathbf{I}_{N}$ is the identity matrix of size $N, \boldsymbol{U}_{f}=\mathbf{I}_{N} \otimes \boldsymbol{e}_{1}$, where $\boldsymbol{e}_{1}$ is the first row of $\mathbf{I}_{Q+1}$, and $\boldsymbol{M}$ is an $N(Q+1) \times N$ matrix given in (A.14) in Appendix A.

Proof: See Appendix A.

Note that the vector $\boldsymbol{I}_{n}$ contains samples of the (continuous) current distribution in (2.10). Using the even symmetry of the dipoles, it suffices to consider vectors of dimension $(Q+1)$ rather than $2 Q+1[14]$. 
From the expression of the matrix $\boldsymbol{M}$ in Appendix A, it can be seen that this matrix incorporates the coupling effects between the dipoles and depends solely on the intrinsic parameters of the array, i.e., its geometry and dimensions of the dipoles, but does not depend on its extrinsic parameters, i.e., excitation voltages and load impedances.

The expression in (2.14) renders the computation of the current distribution vector, $\boldsymbol{I}$, and subsequently the optimization of the array gain highly efficient. To see this, we note that the optimization of the array gain will require varying the load reactances and excitation voltages, but will not change the intrinsic parameters of the array. Since the matrix $\boldsymbol{M}$ does not depend on the load reactances and excitation voltages, it is evaluated only once prior to optimization. Furthermore, we note that evaluating the current distribution through the expressions in (2.14) for any excitation vector $\boldsymbol{V}$ and load reactance vector $\boldsymbol{X}$ is more computation attractive than evaluating it using the expression in [14]. To see that, we note that the number of samples, $Q+1$, is typically much greater than the number of dipole elements, i.e., $Q+1 \gg N$. Now, computing the vector $\boldsymbol{I}$ requires inverting the $N \times N$ matrix $\mathbf{I}_{N}+\jmath \operatorname{diag}\{\boldsymbol{X}\} \boldsymbol{U}_{f} \boldsymbol{M}$ which is significantly less computationally demanding than the corresponding expressions in [14], which require inverting a $N(Q+1) \times N(Q+1)$ matrix with entries that depend on both the intrinsic and extrinsic parameters of the array.

Having described the methodology of obtaining the exact current distributions, we briefly present the sinusoidal approximation of the current distribution. 


\subsubsection{The Sinusoidal Approximation of the Current Distri- bution}

One of the main difficulties of obtaining the exact current distribution arises from the intricacy of the thin-wire kernel in (2.11). To avoid this difficulty, this kernel is replaced with a Dirac delta function, which gives rise to a sinusoidal current distribution on the dipole elements. This approach simplifies the expression of the current distribution on the antenna elements, and facilitates the computation of load reactances needed to achieve approximate beam patterns $[1,5,11]$. However, this approximation can result in beam patterns that are significantly different from those yielded by the load reactances obtained using exact current distributions as elucidated below. The details of obtaining this approximation from the Hallen equations in (2.10) is summarized in Appendix B and the final result is given in the following remark.

Remark 1. For any excitation voltages vector, $\boldsymbol{V}$, and load reactances vector, $\boldsymbol{X}$, the sinusoidal approximation of the current distribution vector $\tilde{\boldsymbol{I}}$ corresponding to Dirac delta thin-wire kernel can be expressed as

$$
\tilde{\boldsymbol{I}}=\tilde{\boldsymbol{M}}\left(\mathbf{I}_{N}+\jmath \operatorname{diag}\{\boldsymbol{X}\} \boldsymbol{U}_{f} \tilde{\boldsymbol{M}}\right)^{-1} \boldsymbol{V}
$$

where $\tilde{\boldsymbol{I}}$ is the vector constructed by stacking the $N$ vectors $\left\{\tilde{\boldsymbol{I}}_{n}\right\}_{n=0}^{N-1}$ corresponding to the $N$ dipoles on top of each other, $\tilde{\boldsymbol{M}}$ is given in (B.16) in Appendix B, and $\boldsymbol{U}_{f}$ is defined in Lemma 2.

Proof: See Appendix B.

Analogous to $\boldsymbol{M}$, the matrix $\tilde{\boldsymbol{M}}$ incorporates the coupling effects under the sinusoidal approximation, depending solely on the geometry of the array and the dimensions of the dipoles. 
The analogy between (2.14) and (2.15) facilitates the comparison between the exact current distribution $\boldsymbol{I}$ and the approximate sinusoidal distribution $\tilde{\boldsymbol{I}}$, which will be discussed in the next section.

\subsubsection{Comparison Between The Exact and Approximate Cur- rent Distributions}

To quantify the error arising from the sinusoidal approximation, we consider the root mean square error (RMSE) between the actual current distribution vector, $\boldsymbol{I}$,

obtained in (2.14) using the MoM, and the sinusoidal current vector, $\tilde{\boldsymbol{I}}$, whose entries are given in (2.15). This RMSE can be expressed as

$$
\mathcal{E}_{\mathrm{I}}=\frac{\|\boldsymbol{I}-\tilde{\boldsymbol{I}}\|}{\|\boldsymbol{I}\|}=\frac{\|(\boldsymbol{T}-\tilde{\boldsymbol{T}}) \boldsymbol{V}\|}{\|\boldsymbol{T} \boldsymbol{V}\|},
$$

where, cf. (2.14) and (2.15),

$$
\begin{aligned}
& \boldsymbol{T}=\boldsymbol{M}\left(\mathbf{I}_{N}+\jmath \operatorname{diag}\{\boldsymbol{X}\} \boldsymbol{U}_{f} \boldsymbol{M}\right)^{-1}, \\
& \tilde{\boldsymbol{T}}=\tilde{\boldsymbol{M}}\left(\mathbf{I}_{N}+\jmath \operatorname{diag}\{\boldsymbol{X}\} \boldsymbol{U}_{f} \tilde{\boldsymbol{M}}\right)^{-1} .
\end{aligned}
$$

The expression in (2.16) provides a quantitative measure of the error incurred by the commonly-used sinusoidal approximation. This expression exposes the dependency of the error on the intrinsic and extrinsic parameters of the array. This error manifests itself in the difference between the matrices $\boldsymbol{T}$ and $\tilde{\boldsymbol{T}}$, which correspond to the equivalent admittances that map $\boldsymbol{V}$ to the respective current distributions.

In the next section, we will expose the role of $\boldsymbol{T}$ and $\tilde{\boldsymbol{T}}$ in the power gains corresponding to the actual and approximate current distributions, which will enable us to develop a framework for obtaining the load impedances and excitation voltages that maximize the power gain in any desired direction. 


\subsection{Calculation of the Power Gain}

To obtain a wieldy expression for the power gain, we substitute the expansion of the current distribution, given in (A.1), from Appendix A, in (2.9), which yields

$$
G_{a}(\phi \mid \boldsymbol{V}, \boldsymbol{X}) \approx \frac{\eta(k \Delta)^{2}}{4 \pi} \frac{\left|\sum_{n=0}^{N-1} \sum_{q=-Q}^{Q} a_{n}^{*}(\phi) I_{n}\left(z_{q}\right)\right|^{2}}{\Re\left\{\sum_{n=0}^{N-1} V_{n}^{*} I_{n}\left(z_{0}\right)\right\}},
$$

where $\Delta$ is the step size defined in (A.3) in Appendix A. Note that the error in this approximation goes to zero as the number of samples $Q$ increases, i.e., $\Delta \rightarrow 0$.

\subsubsection{The Power Gain as a Rayleigh Quotient: Exact}

Using the expression of the exact current distribution in (2.14), the gain expression in (2.19) can be expressed in a compact form using vector notation, directly in terms of the excitation voltages $\boldsymbol{V}$. In particular, we have the following lemma.

Lemma 3. Given the excitation voltages vector, $\boldsymbol{V}$, and load reactances vector, $\boldsymbol{X}$, the azimuth power gain $G_{a}(\phi \mid \boldsymbol{V}, \boldsymbol{X})$ can be expressed as a Rayleigh Quotient

$$
G_{a}(\phi \mid \boldsymbol{V}, \boldsymbol{X})=\frac{\boldsymbol{V}^{\dagger} \boldsymbol{A}(\phi) \boldsymbol{V}}{\boldsymbol{V}^{\dagger} \boldsymbol{B} \boldsymbol{V}}
$$

where the $N \times N$ matrices $\boldsymbol{A}$ and $\boldsymbol{B}$ are given in (C.6) in Appendix A. Proof: See Appendix $C$

For any angle $\phi$, the matrix $\boldsymbol{A}(\phi)$ is rank 1 positive semi-definite, and since $G_{a}(\phi \mid \boldsymbol{V}, \boldsymbol{X})$ is finite and non-negative, it follows that the matrix $\boldsymbol{B}$ is positive definite.

\subsubsection{The Power Gain as a Rayleigh Quotient: Approximate}

Using a derivation analogous to the one give in the previous section, it can be seen that, to obtain the power gain $\tilde{G}_{a}$ under the sinusoidal approximation, the matrix $\boldsymbol{T}$ 
in the definition of the matrices $\boldsymbol{A}$ and $\boldsymbol{B}$ in (C.6) is replaced with its approximated counterpart, $\tilde{\boldsymbol{T}}$.

\subsubsection{The Power Gain as a Weighted Pattern: Exact}

It is often convenient to express the power gain in the form of complex weights multiplied by the steering vector.

Remark 2. The gain in (2.20) can be expressed as

$$
G_{a}(\phi \mid \boldsymbol{V}, \boldsymbol{X})=\left|\boldsymbol{w}^{\dagger} \boldsymbol{a}(\phi)\right|^{2}
$$

where the weights vector $\boldsymbol{w}$ is given by

$$
w=\frac{U_{s} T V}{\sqrt{V^{\dagger} B V}} .
$$

The form in (2.21) is commonly used in modelling beam patterns. In the case of active antenna arrays, the weights, $\boldsymbol{w}$, are complex multipliers, and their optimization to yield beam patterns with particular properties can, in several cases, be cast in a convex form [17]. In contrast, for antenna arrays with passive elements, the dependency of the weights on the load impedances is given in (2.22). Unfortunately, this dependency results in highly non-convex problems. To alleviate this difficulty, in Section 2.4 we will propose an efficient technique to optimize $G_{a}(\phi \mid \boldsymbol{V}, \boldsymbol{X})$.

\subsubsection{The Power Gain as a Weighted Pattern: Approximate}

For the sinusoidal current distribution, the approximate power gain expression $\tilde{G}_{a}$ can expressed in a form analogous to the one in $(2.21)$, but with the matrix $\boldsymbol{T}$ replaced with $\tilde{\boldsymbol{T}}$ yielding a corresponding weight vector $\tilde{\boldsymbol{w}}$. 


\subsubsection{Comparison Between the Power Gain of the Exact and Sinusoidal Approximation Current Distributions}

From the gain expression in (2.21), it can be readily verified that the difference between the exact gain, $G_{a}(\phi \mid \boldsymbol{V}, \boldsymbol{X})$ and that obtained under the sinusoidal approx-

imation, $\tilde{G}_{a}(\phi \mid \boldsymbol{V}, \boldsymbol{X})$, depends solely on the matrices $\boldsymbol{T}$ and $\tilde{\boldsymbol{T}}$, and subsequently on the weights, $\boldsymbol{w}$ and $\tilde{\boldsymbol{w}}$, respectively.

Using the gain expressions at any direction of interest, $\phi_{0}$, the error between the exact and approximate power gains for a given array geometry, excitation voltage vector, $\boldsymbol{V}$, and a load reactance vector, $\boldsymbol{X}$, can be expressed as

$$
\mathcal{E}_{\mathrm{G}}=\left|1-\frac{\tilde{G}_{a}\left(\phi_{0} \mid \boldsymbol{V}, \boldsymbol{X}\right)}{G_{a}\left(\phi_{0} \mid \boldsymbol{V}, \boldsymbol{X}\right)}\right|=\left.|1-| \frac{\tilde{\boldsymbol{w}}^{\dagger} \boldsymbol{a}\left(\phi_{0}\right)}{\boldsymbol{w}^{\dagger} \boldsymbol{a}\left(\phi_{0}\right)}\right|^{2} \mid .
$$

In the numerical results section, we will show that this error can be significant. In particular, we will show that in the case of the Harrington array presented in Fig. 3.1, the load reactance vector that maximizes $G_{a}\left(\phi_{0} \mid \boldsymbol{V}, \boldsymbol{X}\right)$ can vary significantly from the one that maximizes $\tilde{G}_{a}\left(\phi_{0} \mid \boldsymbol{V}, \boldsymbol{X}\right)$.

In the next section, we will develop a technique for determining the excitation voltages of the active elements and the load reactances for the passive elements that maximize the gain for a desired direction $\phi_{0}$.

\subsection{Power Gain Optimization}

In this section we consider the optimization of the excitation voltages and the load reactances in order to maximize the power gain at a given azimuth angle $\phi_{0}$. The optimization of the power gain of antenna arrays with passive elements is more intricate than that of its all-active counterpart. Furthermore, although this can be viewed as the maximization of the power delivered to the medium in a given direction, 
the application of the maximum power transfer theorem would require cumbersome simplification of the power gain expression. Thus, our goal in this section is to propose an algorithm to solve the following optimization problem:

$$
\begin{array}{ll}
\underset{\boldsymbol{V}, \boldsymbol{X}}{\operatorname{maximize}:} & G_{a}\left(\phi_{0} \mid \boldsymbol{V}, \boldsymbol{X}\right), \\
\text { subject to : } & V_{n}=0, \quad n \in \mathcal{N}_{p}, \\
& X_{n}=0, \quad n \in \mathcal{N}_{a} .
\end{array}
$$

From (2.20), it can be seen that the gain, $G_{a}\left(\phi_{0} \mid \boldsymbol{V}, \boldsymbol{X}\right)$, depends on the reactances, $\boldsymbol{X}$, through the $\boldsymbol{T}$ matrix defined in (2.17). From these expressions, it can be readily verified that $G_{a}\left(\phi_{0} \mid \boldsymbol{V}, \boldsymbol{X}\right)$ is not a convex function in $\boldsymbol{X}$, which renders its optimization rather difficult. To circumvent this difficulty, in this section we will provide an approach which tends to yield close-to-optimal solutions.

The non-convexity of the the gain function, $G_{a}\left(\phi_{0} \mid \boldsymbol{V}, \boldsymbol{X}\right)$, implies that the solution reached by a standard solver will depend primarily on the initial point. Hence, determining a 'good' initial point is essential for the algorithm to avoid being trapped at local maxima or saddle points. Towards alleviating the potential of such occurrences, we begin by making the following two observations. First, the gain in (2.20) is invariant to scaling of the excitation voltage, i.e., for any constant $c, G_{a}\left(\phi_{0} \mid c \boldsymbol{V}, \boldsymbol{X}\right)=$ $G_{a}\left(\phi_{0} \mid \boldsymbol{V}, \boldsymbol{X}\right)$. Hence, it suffices to consider unit-norm excitation voltage vectors. Second, we note that the gain achieved by an antenna array with passive elements must be upper bounded by the gain achieved by its all-active counterpart. Using this observation, we will find the optimal excitation voltages required to achieve a maximum gain in a given direction $\phi_{0}$, assuming that all elements are active with zero load reactances. Finding the globally optimal excitation voltages vector is straightforward, as will be elaborated below. 
In general, in an antenna array, only a subset of elements is active, whereas the rest of the elements are passive. For instance, in the Harrington array considered in Section 3, only the first (central) element of the array is active. Hence, upon convergence of the algorithm, only the entries of the excitation voltage vector corresponding to the active elements in the array must be non-zero. In other words, for the Harrington array example, the excitation voltage vector is $\boldsymbol{V}=\left[\begin{array}{llll}1 & 0 & \cdots & 0\end{array}\right]^{\top}$. For general arrays, the optimal excitation voltages vector is given in the following section.

\subsubsection{An Efficient Algorithm for Optimizing the Power Gain of Antenna Arrays With Active and Passive Elements}

The philosophy of the algorithm presented herein is based on the observation that the optimum power gain of active antenna arrays upper bounds that of passive ones. We proceed iteratively as follows. Initially, we find the optimal excitation voltages vector assuming all the antennas are active with zero load reactances. We also find a destination vector which corresponds to the optimal excitation voltages when only the elements in $\mathcal{N}_{a}$ are active and the elements in $\mathcal{N}_{p}$ are passive with zero load reactances. The initial vector corresponding to the all-active antenna array is slightly rotated towards the destination vector that maximizes the gain of the antenna array with zero load reactances. Next, the, potentially local, optimal load reactances for the elements in $\mathcal{N}_{p}$ corresponding to the rotated excitation voltages vector is computed using a steepest ascent algorithm. For the obtained set of reactances, the optimum entries of the excitation voltage vector, corresponding to only the elements in $\mathcal{N}_{a}$ are recomputed; the remaining entries of the excitation voltages vector are zero. The destination excitation voltage vector is updated and the current excitation voltages vector is rotated towards it. This process is iteratively carried out until 
the distance between the current and destination excitation voltages falls below a prescribed threshold. The steps of the proposed algorithm are as follows.

\section{Initial Excitation Voltages Vector}

To find the initial point of the proposed algorithm, we assume that all the elements of the array are active with zero load reactances. The optimal excitation voltages vector, $\hat{\boldsymbol{V}}^{*}$, that maximizes the gain in the direction of $\phi_{0}$ for this array is given by

$$
\hat{\boldsymbol{V}}^{*}=\arg \max _{\boldsymbol{V}} G_{a}\left(\phi_{0} \mid \boldsymbol{V}, \mathbf{0}\right)
$$

The solution of (2.25) is readily given in closed form as

$$
\hat{\boldsymbol{V}}^{*}=\frac{\boldsymbol{B}^{-1} \boldsymbol{\alpha}\left(\phi_{0}\right)}{\left\|\boldsymbol{B}^{-1} \boldsymbol{\alpha}\left(\phi_{0}\right)\right\|} .
$$

\section{Computing the Destination Excitation Vector}

To find the destination excitation voltages vector corresponding to the case in which the passive elements in $\mathcal{N}_{p}$ are terminated with a given set of load reactances, we partition the excitation voltage vector, $\boldsymbol{V}$, and the load reactance vector, $\boldsymbol{X}$, into $\left|\mathcal{N}_{a}\right|$-dimensional vectors $\boldsymbol{V}_{a}$ and $\boldsymbol{X}_{a}$, respectively, corresponding to the active

elements, and $\left|\mathcal{N}_{p}\right|$-dimensional vectors $\boldsymbol{V}_{p}$ and $\boldsymbol{X}_{p}$, respectively, corresponding to the passive elements, i.e.,

$$
\boldsymbol{V}=\left[\begin{array}{l}
\boldsymbol{V}_{a} \\
\boldsymbol{V}_{p}
\end{array}\right], \quad \boldsymbol{X}=\left[\begin{array}{l}
\boldsymbol{X}_{a} \\
\boldsymbol{X}_{p}
\end{array}\right]
$$

At the destination excitation voltage vector, the reactances corresponding to the active elements must be zero, i.e., $\boldsymbol{X}_{a}=\mathbf{0}$, and the excitation voltages corresponding to the passive elements must also be zero, i.e., $\boldsymbol{V}_{p}=\mathbf{0}$. Given that the passive 
elements are terminated with load reactances $\boldsymbol{X}_{p}$, the optimum excitation voltages for the active elements, $\boldsymbol{V}_{a}^{*}$, in the destination vector can be expressed as

$$
\boldsymbol{V}_{a}^{*}=\arg \max _{\boldsymbol{V}_{a}} G_{a}\left(\phi_{0} \mid\left[\boldsymbol{V}_{a}^{\top} \mathbf{0}^{\top}\right]^{\top},\left[\begin{array}{ll}
\mathbf{0}^{\top} & \boldsymbol{X}_{p}^{\top}
\end{array}\right]^{\top}\right)
$$

Similar to (2.26), the excitation voltages corresponding to the active elements, obtained from solving (2.28), can be expressed in a closed form as follows:

$$
\boldsymbol{V}_{a}^{*}=\frac{\boldsymbol{B}_{a}^{-1} \boldsymbol{\alpha}_{a}\left(\phi_{0}\right)}{\left\|\boldsymbol{B}_{a}^{-1} \boldsymbol{\alpha}_{a}\left(\phi_{0}\right)\right\|},
$$

where $\boldsymbol{B}_{a}$ and $\boldsymbol{\alpha}_{a}$ are the partitions of $\boldsymbol{B}$ and $\boldsymbol{\alpha}$ corresponding to the active elements

$$
\begin{array}{r}
\boldsymbol{B}_{a}=\left[B_{m, n}\right], m, n \in \mathcal{N}_{a}, \\
\boldsymbol{\alpha}_{a}=\left[\alpha_{n}\right], n \in \mathcal{N}_{a} .
\end{array}
$$

This yields an optimal excitation voltage vector in the form

$$
\boldsymbol{V}^{*}=\left[\begin{array}{c}
\boldsymbol{V}_{a}^{*} \\
\mathbf{0}
\end{array}\right] \text {. }
$$

\section{Rotating the Excitation Voltage Vector}

We now describe the process of rotating a voltage vector $\hat{\boldsymbol{V}}$ in the direction of a destination voltage vector $\boldsymbol{V}^{*}$, to obtain a voltage vector $\boldsymbol{V}$. For convenience, we will express each entry of the excitation voltage vectors using the polar coordinates, that is, $V_{n}=v_{n} e^{\jmath \psi_{n}}$, where $v_{n}=\left|V_{n}\right|$. Based on the scaling invariance of the array gain, we will consider unit norm excitation voltages satisfying $\sum_{n=0}^{N-1} v_{n}^{2}=1$. Such an $N$-dimensional vector can be expressed in the $(N-1)$-dimensional polar coordinates explicitly given in (D.2) in Appendix D. Using this representation, the excitation voltage vector, $\boldsymbol{V}$, will be written as $\boldsymbol{V}(\boldsymbol{\theta}, \boldsymbol{\psi})$, where $\boldsymbol{\theta}$ is the vector containing the $N-1$ angles in the polar representation of the vector containing the magnitudes $\left\{v_{n}\right\}_{n=0}^{N-1}$ and $\boldsymbol{\psi}$ is the vector containing the phases of $\boldsymbol{V}$. 
At any iteration, let $\hat{\boldsymbol{V}}(\hat{\boldsymbol{\theta}}, \hat{\boldsymbol{\psi}})$ be the source of rotation, while $\boldsymbol{V}(\boldsymbol{\theta}, \boldsymbol{\psi})$ be the rotated vector towards the destination vector $\boldsymbol{V}^{*}\left(\boldsymbol{\theta}^{*}, \boldsymbol{\psi}^{*}\right)$. Then for any $\gamma \in[0,1]$, the rotated vector $\boldsymbol{V}$ can be expressed as

$$
\boldsymbol{V}=\hat{\boldsymbol{V}}(\hat{\boldsymbol{\theta}}+\gamma \boldsymbol{\Delta} \boldsymbol{\theta}, \hat{\boldsymbol{\psi}}+\gamma \boldsymbol{\Delta} \boldsymbol{\psi})
$$

where $\boldsymbol{\Delta} \boldsymbol{\theta}=\boldsymbol{\theta}^{*}-\hat{\boldsymbol{\theta}}$, and $\boldsymbol{\Delta} \boldsymbol{\psi}=\boldsymbol{\psi}^{*}-\hat{\boldsymbol{\psi}}$.

Note that using the polar representation of the vector containing the magnitudes $\left\{v_{n}\right\}_{n=0}^{N-1}$ ensures that the construction in (2.32) satisfies $\|\boldsymbol{V}\|=1$. In this construction, we note that the phases of the destination excitation voltages corresponding to the passive elements are chosen to be equal to those of the initial excitation.

\section{Optimization of the Reactances of the Passive Elements}

Given the excitation voltages vector $\boldsymbol{V}$ generated in the previous step, we optimize the load reactances vector, $\boldsymbol{X}_{p}$. In particular, we wish to solve the following, unfortunately non-convex, optimization problem

$$
\boldsymbol{X}_{p}^{*}=\arg \max _{\boldsymbol{X}_{p}} G_{a}\left(\phi_{0} \mid \boldsymbol{V},\left[\mathbf{0}^{\top} \boldsymbol{X}_{p}^{\top}\right]^{\top}\right)
$$

To obtain a solution of (2.33), we use the steepest ascent algorithm. At the $i$-th iteration of this algorithm, the reactances are updated as follows:

$$
\boldsymbol{X}_{p_{i+1}}^{*}=\boldsymbol{X}_{p_{i}}^{*}+\mu \nabla_{\boldsymbol{X}_{p}} G_{a}\left(\phi_{0} \mid \boldsymbol{V}, \boldsymbol{X}_{p_{i}}^{*}\right)
$$

where $\mu$ is the step size and $\nabla_{\boldsymbol{X}_{p}} G_{a}$ is the gradient of the power gain with respect to the load reactances of the passive elements. For convenience, this gradient is given explicitly in Appendix E. Iterations continue until convergence.

The reactances at the 0 -th iteration of the steepest ascent algorithm, $\boldsymbol{X}_{p_{0}}^{*}$, are chosen to be those obtained from the previous rotation of the excitation voltages 
vector. The proposed algorithm is summarized in the following flow chart in Fig. 2.4.

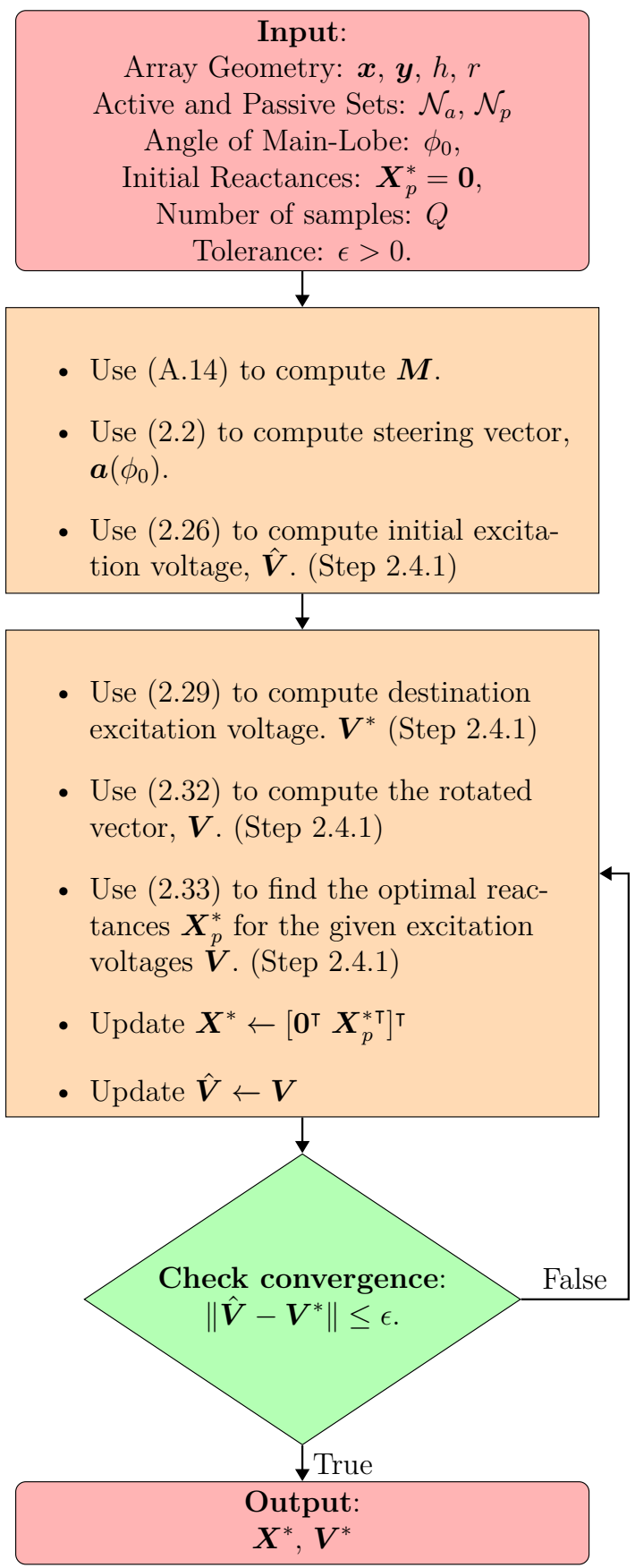

Figure 2.4: Flowchart of the optimization algorithm in Section 2.4. 


\section{Chapter 3}

\section{Numerical Results}

In this chapter, we first consider the case of 1 active element and 1 passive element to study the effect of the load reactance on the error between the sinusoidal current distribution and the exact one. Then, we consider the Harrington array described in Chapter 2.1, when the dipoles are half wavelength, i.e., $h=\frac{\lambda}{4}$, of radius $r=\frac{h}{100} \lambda$ each, the number of array elements is $N=7$ and the radius of the array is $R=\frac{\lambda}{4}$. We show that the power gain pattern obtained using the reactances given in [1] mismatches the expected pattern based on the sinusoidal approximation of the current distribution. We also consider a multiple-active multiple-passive circular array with 3 active elements and 9 passive elements as shown in Chapter 2.

An example of such an array is the Harrington array shown in Fig. 3.1 [1], which will be used to validate the analysis and the methodology proposed hereinafter. In the Harrington array, an active element is excited by a voltage source positioned at the origin and surrounded by $N-1$ passive elements. The passive elements are terminated with reactive loads and placed uniformly around a circle of radius $R$, cf. Fig. 3.1. The locations of the passive elements are given by $x_{n}=R \cos \left((n-1) \frac{2 \pi}{N-1}\right)$ and $y_{n}=R \sin \left((n-1) \frac{2 \pi}{N-1}\right), n=1, \ldots, N-1$. 


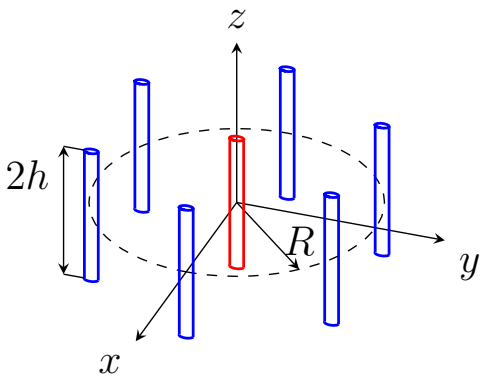

Figure 3.1: A Harrington array with $N=7$ dipoles.

We consider an example of an antenna array with multiple-active and multiplepassive elements. In this array, 3 active elements are excited by voltage sources and positioned uniformly around a circle of radius $\frac{\lambda}{4}$ as shown in Fig. 3.2, which are all surrounded by 9-passive elements terminated with reactive loads and placed uniformly

around a circle of radius $\frac{\lambda}{2}$. The locations of the active and passive elements are given by

$$
\begin{aligned}
& x_{n}=\frac{\lambda}{4} \cos \left((n-1) \frac{2 \pi}{3}\right) \\
& y_{n}=\frac{\lambda}{4} \sin \left((n-1) \frac{2 \pi}{3}\right), \quad n=0,1,2,
\end{aligned}
$$

and

$$
\begin{aligned}
& x_{n}=\frac{\lambda}{2} \cos \left((n-3) \frac{2 \pi}{9}\right) \\
& y_{n}=\frac{\lambda}{2} \sin \left((n-3) \frac{2 \pi}{9}\right), \quad n=3, \ldots, 11
\end{aligned}
$$

respectively.

\subsection{Error in Sinusoidal Current Distribution for an Array of two Elements}

In this example, we investigate the error between the sinusoidal approximation of the current distribution and the exact for an array of two dipoles with one being active and the other being passive with a variable load reactance. 


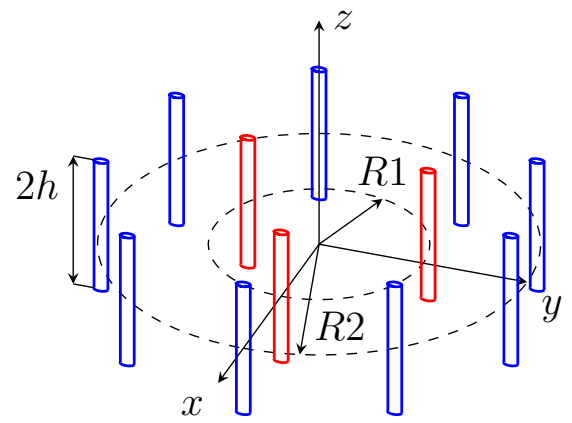

Figure 3.2: Uniform circular array with 3 active and 9 passive elements.

For the error in the current distribution, $\mathcal{E}_{I}$, we evaluate $(2.16)$, which is depicted in Fig. 3.3.

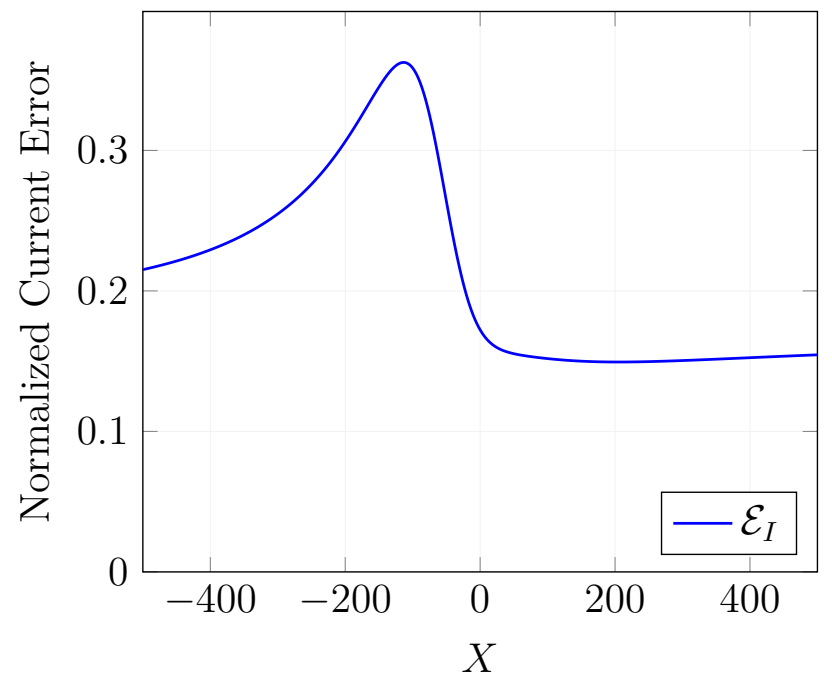

Figure 3.3: Current error for an array composed of 1 active and 1 passive, for load reactance $X$.

From Fig. 3.3, it can be seen that the error between the sinusoidal current distribution and the exact one can be significant. For instance, this error is at least more than $10 \%$ and is even above $30 \%$ when $-200 \leq X \leq-50$. Our numerical experiments 
suggest that this error can be significantly larger for other configurations and load reactances.

\subsection{Error in the Power Gain Pattern of the Harrington Array}

In this example, we compare the power gain patterns for the Harrington array for the load reactances provided in [1], obtained under the sinusoidal approximation of current distribution, and the expected power gain pattern. Four cases are considered corresponding to main-lobe angles $\phi_{0}=0^{\circ}, 10^{\circ}, 20^{\circ}, 30^{\circ}$. Note that because of the symmetry of the array, the patterns repeat every $30^{\circ}$.

For each case, we first obtain the expected power gain pattern by evaluating the power gain expression based on the sinusoidal approximation give in $(2.20)$, i.e. $\tilde{G}_{a}$, for the load reactances $\left\{\tilde{X}_{n}^{*}\right\}_{n=1}^{6}$ obtained in [1] and given in Table 3.1. Then, we provide the results obtained by numerically evaluating the analytical gain expression in $(2.20)$ based on the exact current distribution, i.e., $G_{a}$. These results are verified using the NEC simulator. Since the 0 -th element is active, $X_{0}^{*}=0$, for all $\phi_{0}$. The polar plots

Table 3.1: Reactances Obtained Using the Algorithm Presented in [1] for the Considered Harrington Array

\begin{tabular}{c|c|c|c|c}
\hline & \multicolumn{4}{|c}{ Main-lobe direction $\phi_{0}$} \\
$\tilde{X}_{n}^{*}$ & $0^{\circ}$ & $10^{\circ}$ & $20^{\circ}$ & $30^{\circ}$ \\
\hline 1 & -63.6 & -64.3 & -64.7 & -57.4 \\
2 & -94.6 & -85.2 & -71.0 & -57.4 \\
3 & 9.0 & 34.8 & 180.0 & -398.1 \\
4 & 10.8 & 11.4 & 9.7 & 1.7 \\
5 & 9.0 & -1.0 & -4.1 & 1.7 \\
6 & -94.6 & -105.6 & -134.1 & -401.0 \\
\hline
\end{tabular}


of the power gain in the azimuth plane obtained by the numerical evaluation of the power gain in (2.20) for the exact and sinusoidal current distributions, and the NEC simulations, for the reactances $\tilde{\boldsymbol{X}}^{*}$, given in Table 3.1, are depicted in Fig. 3.4.

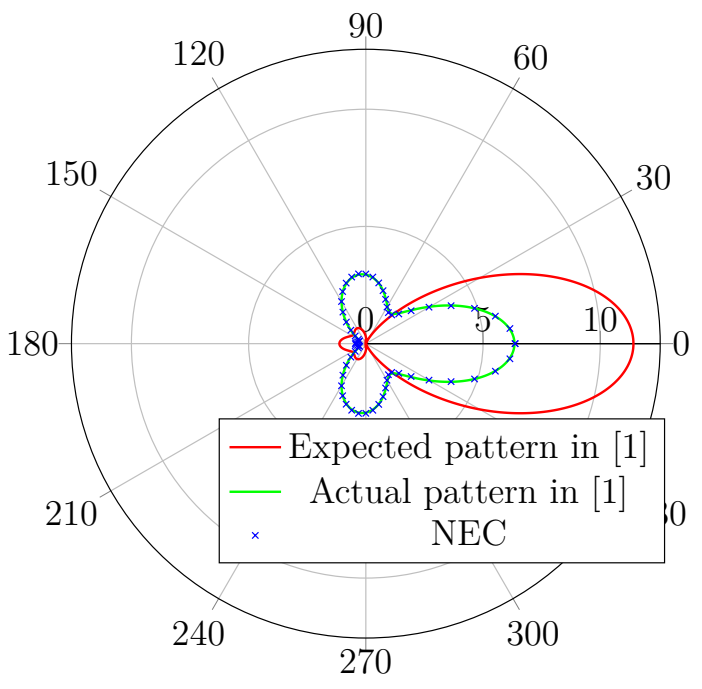

(a) Main-Lobe at $0^{\circ}$

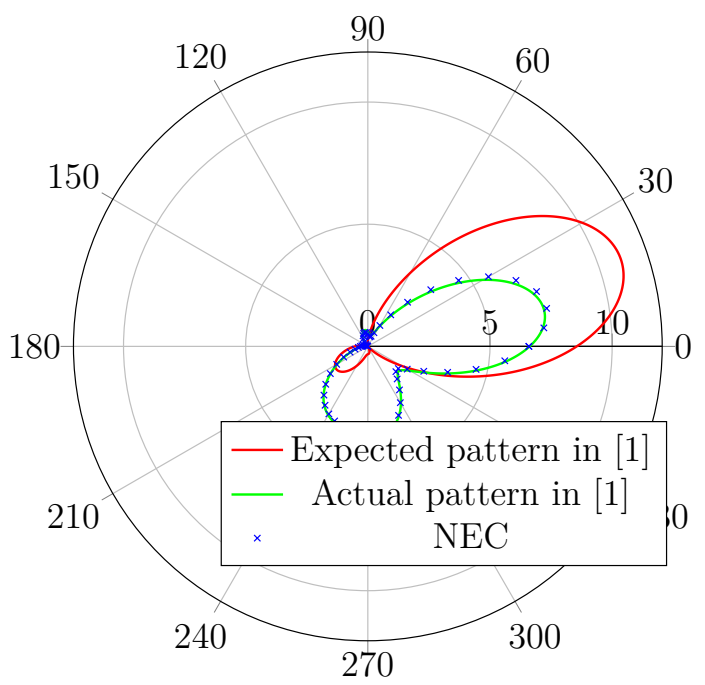

(c) Main-Lobe at $20^{\circ}$

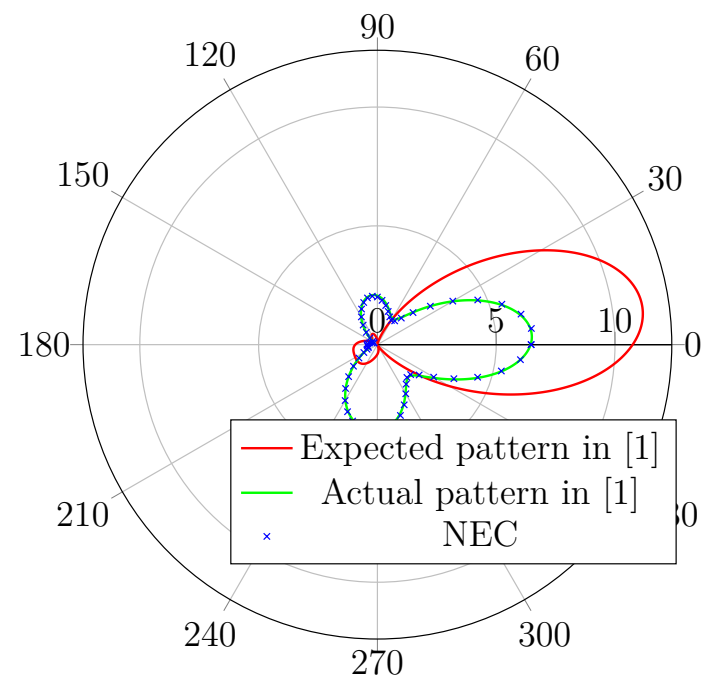

(b) Main-Lobe at $10^{\circ}$

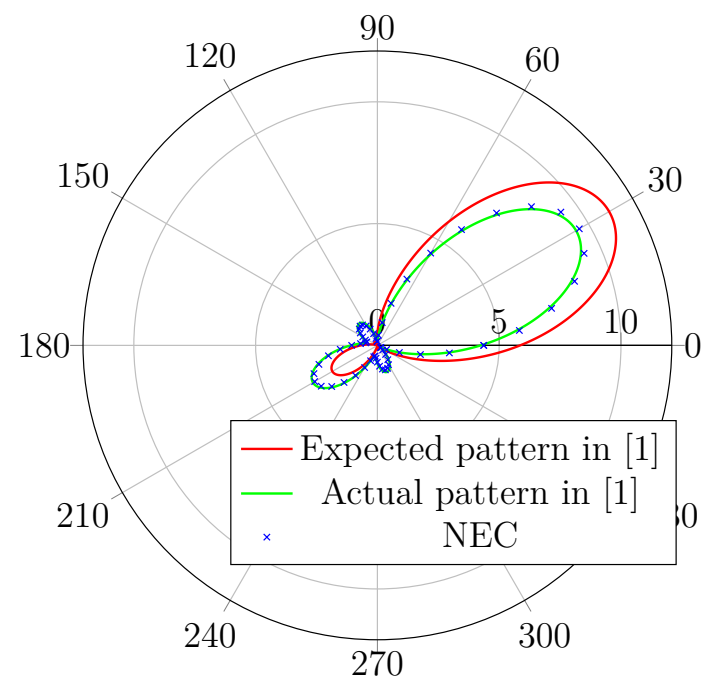

(d) Main-Lobe at $30^{\circ}$

Figure 3.4: Polar plot in the azimuth plane of the expected and actual power gains for beam direction at $\phi_{0}=0^{\circ}, 10^{\circ}, 20^{\circ}, 30^{\circ}$ using the reactances obtained in [1]. 
From Fig. 3.4, it can be seen that the numerical results of the expected power gain patterns, denoted by the red solid lines, do not agree with the exact power gain patterns, denoted by green solid lines, based on the analytical expressions derived herein, which are in agreement with the results obtained from the NEC simulations, denoted by ' $x$ '. More importantly, the obtained power gain patterns have a significantly smaller main-lobe and larger side-lobes. The error in the power gain between the expected pattern and the exact one for the four cases of the main-lobe angle $\phi_{0}=0^{\circ}, 10^{\circ}, 20^{\circ}, 30^{\circ}$, are $44 \%, 43 \%, 35 \%, 13 \%$, respectively. Thus, the results given herein assert the importance of obtaining the exact current distribution whilst performing the optimization routine.

\subsection{Optimization of the Harrington Array}

In this example, we show the power gain patterns for the Harrington array obtained using the optimization algorithm presented in Chapter 2.4. The results are obtained by numerically evaluating the power gain expression given in (2.20), which are verified using the NEC simulations. The reactances obtained from the optimization algorithm and used in the evaluation of the power gain are given in Table 3.2. The polar plots of the power gain patterns are depicted in Fig.3.5.

From Fig. 3.5, it can be seen that the optimized power gain matches the expected one and is verified using the NEC simulation results. This asserts that the power gains achieved by the reactances given in Table 3.2 are significantly higher than 
Table 3.2: Reactances Obtained Using the Algorithm Presented in Chapter 2.4 for the Considered Harrington Array

\begin{tabular}{c|c|c|c|c}
\hline & \multicolumn{4}{|c}{ Main-lobe direction $\phi_{0}$} \\
$X_{n}^{*}$ & $0^{\circ}$ & $10^{\circ}$ & $20^{\circ}$ & $30^{\circ}$ \\
\hline 1 & -88.1 & -88.8 & -89.2 & -84.0 \\
2 & -135.1 & -120.2 & -99.4 & -84.0 \\
3 & 4.1 & 30.2 & 131.6 & -648.2 \\
4 & 3.8 & 6.0 & 5.5 & -7.6 \\
5 & 4.1 & -7.8 & -12.0 & -7.6 \\
6 & -135.1 & -154.4 & -205.1 & -648.2 \\
\hline
\end{tabular}

those achieved by the reactances given in Table 3.1. For example, the power gain achieved when the main-lobe direction is $\phi_{0}=0^{\circ}$ using the reactances in Table 3.2 is 11.479, whereas that obtained using those in Table 3.1 is 6.368. Similar results hold at other main lobe directions. From these results, it can be concluded that using the (exact) Hallen equations along with the MoM technique and the proposed optimization algorithm can yield significant gain advantages over the method based on the sinusoidal approximation.

\subsection{Optimization of a circular array with 3 active and 9 pas- sive elements}

In this example, we present the power gain patterns obtained using the aforementioned optimization algorithm applied to the circular array of 3 active and 9 passive elements defined in Chapter 2.1.1. The power gain patterns are compared with those obtained for the same array configuration with all elements being active. Tables 3.4 and 3.3 provide the optimized reactances for the passive elements and the optimized excitation voltages for the active elements, respectively, which are obtained using the 


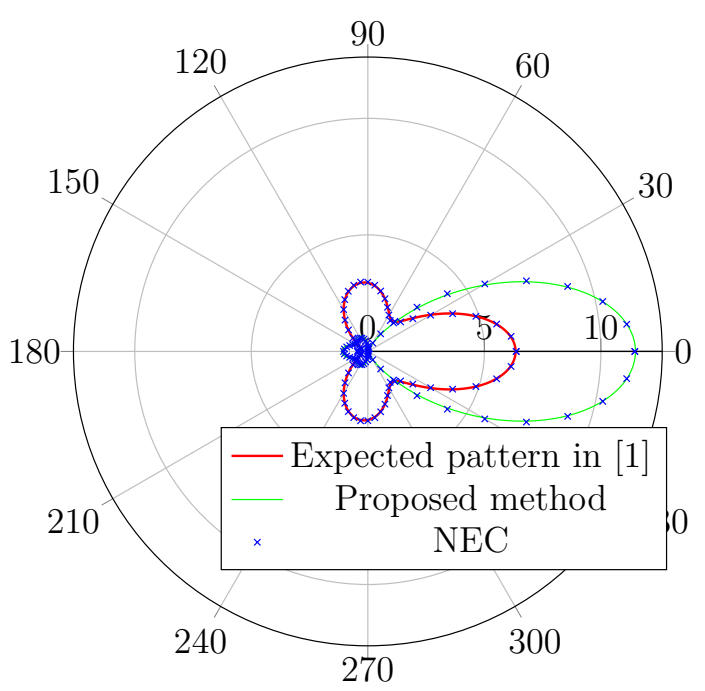

(a) Main-Lobe at $0^{\circ}$

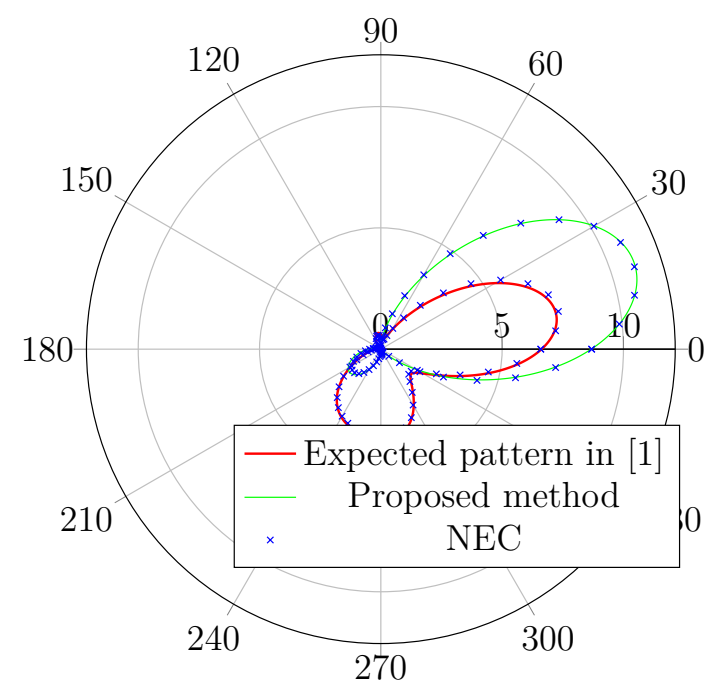

(c) Main-Lobe at $20^{\circ}$

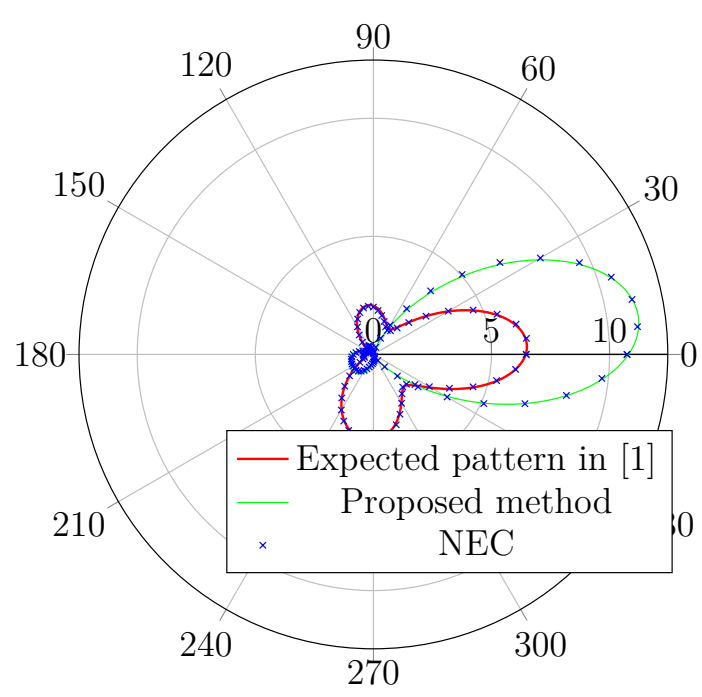

(b) Main-Lobe at $10^{\circ}$

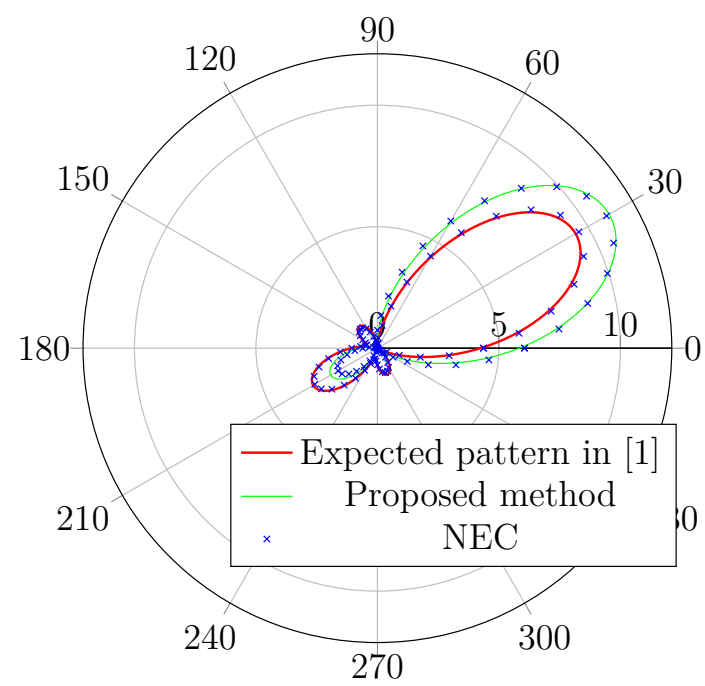

(d) Main-Lobe at $30^{\circ}$

Figure 3.5: Polar plot in the azimuth plane of the expected and actual power gains of the Harrington array, for beam direction at $\phi_{0}=0^{\circ}, 10^{\circ}, 20^{\circ}, 30^{\circ}$ using the reactances obtained from the proposed optimization algorithm.

aforementioned optimization algorithm for four cases, where the main-lobe angles are $\phi_{0}=0^{\circ}, 20^{\circ}, 40^{\circ}, 60^{\circ}$. Due to the symmetry of the array, the patterns repeat every $60^{\circ}$. 
Table 3.3: Voltages Obtained Using the Algorithm Presented in Chapter 2.4 for the Considered 3-active 9-passive Array

\begin{tabular}{|c|c|c|c|c|}
\hline \multirow[b]{2}{*}{$V_{n}^{*}$} & \multicolumn{4}{|c|}{ Main-lobe direction $\phi_{0}$} \\
\hline & $0^{\circ}$ & $20^{\circ}$ & $40^{\circ}$ & $60^{\circ}$ \\
\hline 0 & $0.7110-0.1672 \jmath$ & $0.7482-0.0891 \mathrm{~J}$ & $0.6184+0.1248 \mathrm{\jmath}$ & $0.4723-0.0350 \mathrm{\jmath}$ \\
\hline 1 & $-0.3905+0.2843 \jmath$ & $-0.1050+0.2852 \jmath$ & 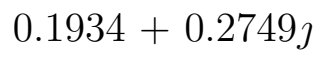 & $0.4723-0.0350 \jmath$ \\
\hline 2 & $-0.3905+0.2841 \jmath$ & $-0.5659+0.1405 \jmath$ & $-0.6950-0.0772 \jmath$ & $-0.7262+0.1552 \jmath$ \\
\hline
\end{tabular}

Table 3.4: Reactances Obtained Using the Algorithm Presented in Chapter 2.4 for the Considered 3-active 9-passive Array

\begin{tabular}{c|c|c|c|c}
\hline & \multicolumn{4}{|c}{ Main-lobe direction $\phi_{0}$} \\
$X_{n}^{*}$ & $0^{\circ}$ & $20^{\circ}$ & $40^{\circ}$ & $60^{\circ}$ \\
\hline 3 & -92.7 & -79.7 & -72.0 & -862.1 \\
4 & -165.9 & -99.0 & -78.8 & -161.7 \\
5 & -124.1 & -197.7 & -136.3 & -161.7 \\
6 & 27.1 & -1.0 & -668.3 & -862.1 \\
7 & -13.5 & -28.6 & -18.8 & -65.0 \\
8 & -13.4 & 7.4 & -8.2 & -33.4 \\
9 & 27 & -16.7 & -16.1 & 19.6 \\
10 & -124.0 & -187.5 & 728.4 & -33.4 \\
11 & -165.9 & -393.9 & -719.1 & -65.0 \\
\hline
\end{tabular}

The polar plots of the power gain patterns corresponding to these cases are depicted in Fig. 3.6.

From Fig. 3.6, it can be seen that the gain achieved by the passive array, although being less than that of the active array, is comparable to the that of the all active counter-part. Thus, a performance comparable to that of the active antenna arrays 
can be achieved using passive antenna arrays, without the complex synchronized RFchains required for the active elements. 


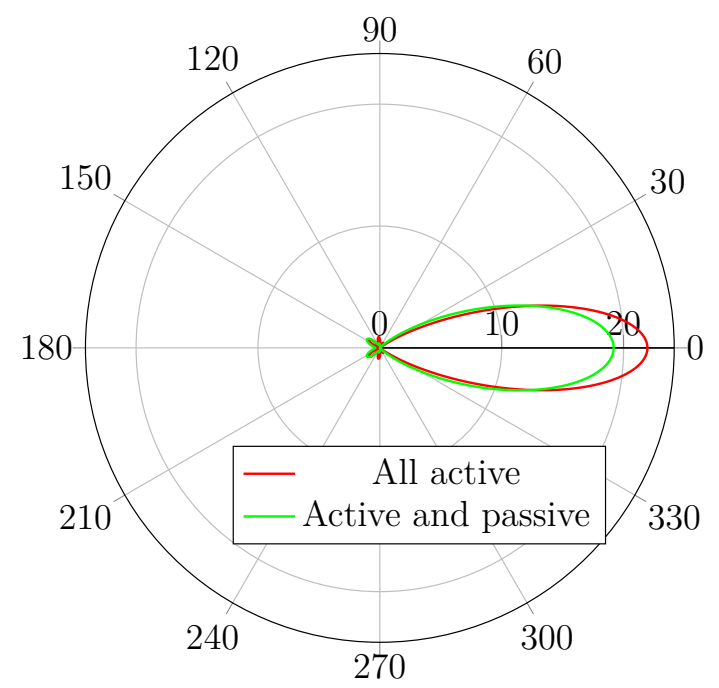

(a) Main-Lobe at $0^{\circ}$

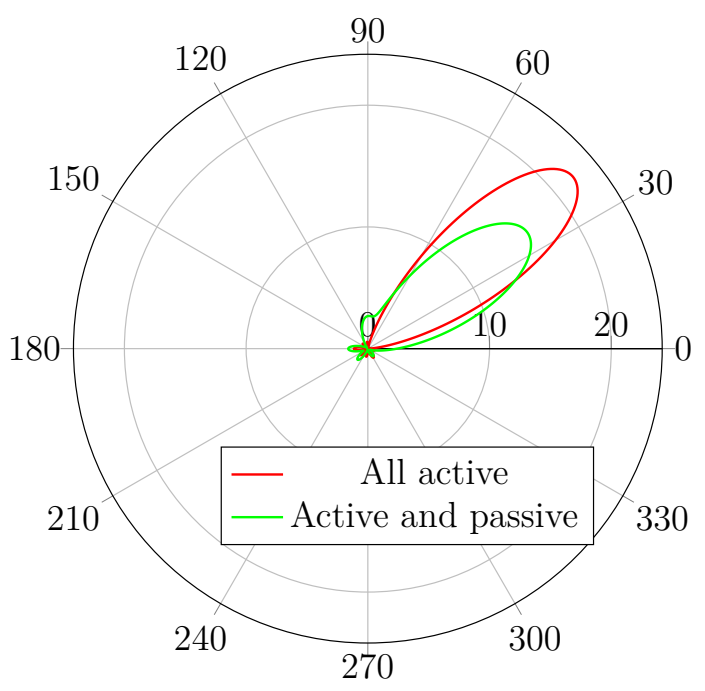

(c) Main-Lobe at $40^{\circ}$

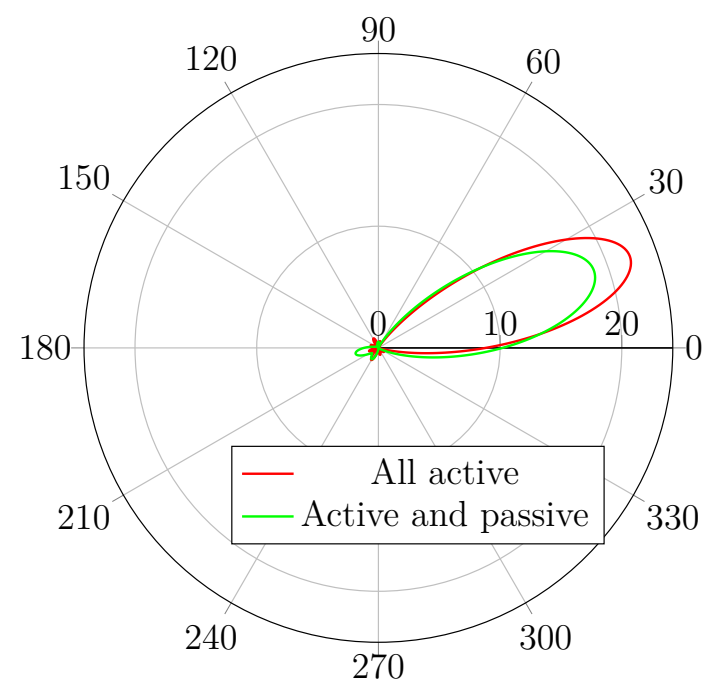

(b) Main-Lobe at $20^{\circ}$

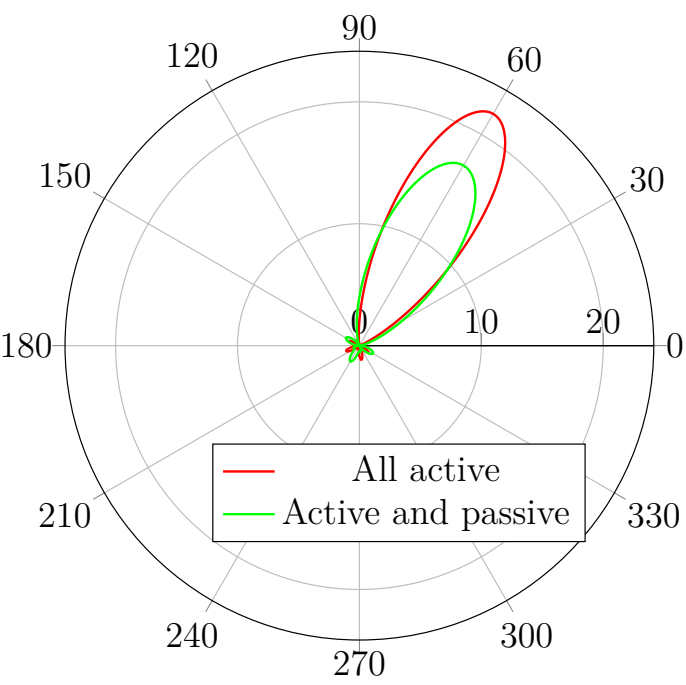

(d) Main-Lobe at $60^{\circ}$

Figure 3.6: Polar plot in the azimuth plane of the expected and actual power gains of the 3 active and 9 passive circular array, for beam direction at $\phi_{0}=0^{\circ}, 20^{\circ}, 40^{\circ}, 60^{\circ}$ using the reactances obtained from the proposed optimization algorithm. 


\section{Chapter 4}

\section{Conclusion and Future Work}

\subsection{Conclusion}

In this work, we considered antenna arrays composed of both active dipole elements excited by voltage sources and reactively controlled passive dipole elements. Towards the end of maximizing the power gain for a given direction, we deviated from the traditional approach in which the current distribution along the antenna elements is assumed to be sinusoidal. Instead, we obtained the exact current distributions by solving the Hallen equations using the MoM technique. Next, we developed an efficient algorithm based on the exact current distributions to maximize the power gain for a given direction. Numerical results show that the power gain patterns obtained based on the sinusoidal approximation yield significantly lower gains than that obtained based on the exact current distributions. The numerical results were verified by the NEC simulation results. Furthermore, it was shown that the power gain achieved by a multiple-active multiple-passive antenna arrays is comparable to that achieved by the same configuration with all elements being active. Which asserts the

appealing value of passive antenna arrays since they require much less complicated feed circuitry. 


\subsection{Future Work}

The analysis in this work can be extended to accommodate 3D monopole or dipole arrays. Moreover, the algorithm proposed herein can be modified to take into account interference and multi-cast beamforming. Furthermore, different array geometries can be investigated to maximize the gain given a constricted space, which would require the optimization of the number of antenna elements and the spacing between them to maximize the gain in a given direction. For more practical calculations, nonideal dipoles and terminating circuits should be considered to incorporate parasitic resistances. Moreover, these computations would be useful in comparisons with experimental results. 


\section{Appendix A}

\section{The Method of Moments (MoM)}

In the MoM technique, the continuous current distribution in (2.10) is approximated with discrete samples thereof. Doing so, transforms the integro-differential equations in (2.10) into a system of linear equations in which the unknowns are the values of the sampled current distribution.

\section{A.1 Basis Function Expansion}

The current distribution, $I_{n}(z)$, along the dipole can be approximated by

$$
I_{n}(z) \approx \sum_{q=-Q}^{Q} I_{n}\left(z_{q}\right) B_{\Delta}\left(z-z_{q}\right), \quad n=1 \ldots, N-1
$$

where the pulse basis-function is given by

$$
B_{\Delta}\left(z-z_{q}\right)= \begin{cases}1, & \left|z-z_{q}\right| \leq \frac{\Delta}{2} \\ 0, & \text { otherwise }\end{cases}
$$

where

$$
z_{q}=q \Delta, \quad \Delta=\frac{2 h}{2 Q+1}, \quad q=-Q \ldots, Q
$$

The accuracy of this approximation depends on the the step $\Delta$, or equivalently, the number of points, $2 Q+1$, at which the current distribution is evaluated. 
For ease of exposition, let $\boldsymbol{I} \in \mathbb{C}^{N(Q+1)}$ be the vector formed by stacking the $N$ the current distribution vectors, $\left\{\boldsymbol{I}_{n}\right\}$, corresponding to the $N$ dipoles. Each of these vectors is of size $Q+1$. In particular,

$$
\boldsymbol{I}=\left[\boldsymbol{I}_{0}^{\top}, \ldots, \boldsymbol{I}_{N-1}^{\top}\right]^{\top}, \quad \boldsymbol{I}_{n}=\left[I_{n}\left(z_{0}\right), \ldots, I_{n}\left(z_{Q}\right)\right]^{\top}
$$

Note that, because of the even symmetry, it is sufficient to find the current samples at the feed point and along half the dipole.

\section{A.1.1 Mutual and Self Impedance Matrices}

Towards finding the current distribution vector $\boldsymbol{I}$, we substitute from (A.1) in (2.10). Rearranging, we obtain a system of linear equations for every dipole $m \in\{0, \ldots, N-$ 1\}. Such a system can be expressed in the following matrix form:

$$
\sum_{n=0}^{N-1} \boldsymbol{Z}_{m, n} \boldsymbol{I}_{n}=C_{m} \boldsymbol{c}_{o}+V_{f_{m}} \boldsymbol{s}_{i}, m=0, \ldots, N-1
$$

where the feed voltage $V_{f_{m}}$ is the voltage at the terminals of the $m$-th dipole given in (2.4). The $(Q+1)$-dimensional vectors $\boldsymbol{c}_{o}$ and $\boldsymbol{s}_{i}$ are given by

$$
\boldsymbol{c}_{\boldsymbol{o}}(q)=\cos \left(k z_{q}\right), \boldsymbol{s}_{\boldsymbol{i}}(q)=\sin \left(k\left|z_{q}\right|\right), \quad q=0, \ldots, Q
$$

The $(Q+1) \times(Q+1)$ matrix $\boldsymbol{Z}_{m, n}$ contains the mutual impedance between the segments of the $m$-th and $n$-th dipoles. In particular, the $p q$-th entry of $\boldsymbol{Z}_{m, n}$ is given by

$$
\begin{aligned}
Z_{m, n}(p, q)=\frac{j \eta}{2 \pi} \int_{-\frac{\Delta}{2}}^{\frac{\Delta}{2}} \frac{1}{1+\delta_{q, 0}} & \left(K_{m, n}\left(z_{p}-u-z_{-q}, d_{m, n}\right)\right. \\
& \left.+K_{m, n}\left(z_{p}-u-z_{q}, d_{m, n}\right)\right) d u, \quad p, q=0, \ldots, Q
\end{aligned}
$$

where $K$ is the (exact) thin-wire kernel in (2.11) and $\delta_{p, 0}$ is the Kronecker delta. 


\section{A.1.2 End of Wire Condition}

To obtain the constants $\left\{C_{m}\right\}_{m=0}^{N-1}$ in (A.5), the condition that $I_{m}\left(z_{Q}\right)=0$ is invoked, which yields

$$
C_{m}=\frac{1}{\boldsymbol{u}_{m}^{\top} \boldsymbol{c}_{o}}\left(\sum_{n \neq m} \boldsymbol{u}_{m}^{\top} \boldsymbol{Z}_{m, n} \boldsymbol{I}_{m}-V_{f_{m}} \boldsymbol{u}_{m}^{\top} \boldsymbol{s}_{i}\right)
$$

where $\boldsymbol{u}_{m} \in \mathbb{C}^{(Q+1)}$ is given by

$$
\boldsymbol{u}_{m}=\boldsymbol{Z}_{m, m}^{-\top} \boldsymbol{u}
$$

and $\boldsymbol{u} \in \mathbb{R}^{(Q+1)}$ is given by $\boldsymbol{u}=[0, \ldots, 0,1]^{\top}$.

Substituting for $C_{m}$ from (A.8) in (A.5) yields

$$
\sum_{n=0}^{N-1} \boldsymbol{Z}_{m, n}^{\prime} \boldsymbol{I}_{n}=V_{f_{m}} \boldsymbol{P}_{m} \boldsymbol{s}_{i} \quad m=0, \ldots, N-1,
$$

where the $(Q+1) \times(Q+1)$ matrices $\left\{\boldsymbol{P}_{m}\right\}_{m=0}^{N-1}$, are

$$
\boldsymbol{P}_{m}=\left(\mathbf{I}_{Q+1}-\frac{\boldsymbol{c}_{o} \boldsymbol{u}_{m}^{\top}}{\boldsymbol{u}_{m}^{\top} \boldsymbol{c}_{o}}\right)
$$

and $\mathbf{I}_{Q+1}$ is the identity matrix of size $Q+1$.

The $N^{2}$ matrices $\left\{\boldsymbol{Z}_{m, n}^{\prime}\right\}_{m, n=0}^{N-1}$ are given by

$$
\boldsymbol{Z}_{m, n}^{\prime}= \begin{cases}\boldsymbol{Z}_{m, m} & m=n, \\ \boldsymbol{P}_{m} \boldsymbol{Z}_{m, n} & m \neq n,\end{cases}
$$

and $Z^{\prime}$ is the block-structured matrix given by

$$
\boldsymbol{Z}^{\prime}=\left[\boldsymbol{Z}_{m, n}^{\prime}\right]_{m, n=0}^{N-1}
$$

\section{A.1.3 Solving for the Current Distribution Vector}

Grouping the $N$ equations in (A.10) in one system of linear equations and left multiplying by $\boldsymbol{Z}^{-1}$ yields

$$
\boldsymbol{I}=\boldsymbol{M} \boldsymbol{V}_{f},
$$


where the $N(Q+1) \times N$ matrix $\boldsymbol{M}$ is given by

$$
\boldsymbol{M}=\left[\boldsymbol{M}_{m, n}\right]_{m, n=0}^{N-1},
$$

and the $(Q+1)$-dimensional vector in this matrix

$$
\boldsymbol{M}_{m, n}=\left(\boldsymbol{Z}^{\prime}\right)_{m, n}^{-1} \boldsymbol{P}_{n} \boldsymbol{s}_{i}, \quad m, n=0, \ldots, N-1
$$

Consequently, from (2.3), the feed current vector $\boldsymbol{I}(0)$ can be expressed as

$$
\boldsymbol{I}(0)=\boldsymbol{U}_{f} \boldsymbol{I}=\boldsymbol{U}_{f} \boldsymbol{M} \boldsymbol{V}_{f},
$$

where $\boldsymbol{U}_{f}$ selects the entries from the current distribution vector corresponding to the feed points. In particular,

$$
\boldsymbol{U}_{f}=\mathbf{I}_{N} \otimes \boldsymbol{e}_{1}
$$

where $\boldsymbol{e}_{1}$ is the first row of $\mathbf{I}_{Q+1}$.

Substituting from (A.15) in (2.4) and solving for $\boldsymbol{V}_{f}$ yields

$$
\boldsymbol{V}_{f}=\left(\mathbf{I}_{N}+\jmath \operatorname{diag}\{\boldsymbol{X}\} \boldsymbol{U}_{f} \boldsymbol{M}\right)^{-1} \boldsymbol{V} .
$$

Substituted for $\boldsymbol{V}_{f}$ in (A.13) yields the following solution of (2.10)

$$
\boldsymbol{I}=\boldsymbol{M}\left(\mathbf{I}_{N}+\jmath \operatorname{diag}\{\boldsymbol{X}\} \boldsymbol{U}_{f} \boldsymbol{M}\right)^{-1} \boldsymbol{V} .
$$

Using (A.18), the sampled current distributions along the dipoles can be obtained for a given set of excitation voltages, load reactances and array geometry. 


\section{Appendix B}

\section{Sinusoidal Approximation of The Current Distribution}

The sinusoidal approximation is usually used to alleviate the complexity of solving the Hallen equations. In this section, we briefly describe the process of obtaining this approximation from the Hallen equations.

\section{B.1 Kernel Approximation as a Scaled Delta Function}

To obtain the sinusoidal approximation of the current distribution from the Hallen equations in $(2.10)$, the kernel, $K(\cdot, \cdot)$, in $(2.10)$ is replaced with a scaled Dirac delta function, yielding the following approximation of the Hallen equations [14]:

$$
\begin{aligned}
\sum_{n=0}^{N-1} \tan (k h) \tilde{Z}_{m, n} \tilde{I}_{n}(z)=\tilde{C}_{m} \cos (k z) & \\
& \quad+\left(V_{m}-\tilde{I}_{m}(0) \jmath X_{m}\right) \sin (k|z|), m=0, \ldots, N-1,
\end{aligned}
$$


where $\tilde{Z}_{m, n}$ represents the mutual impedance between the $m$-th and $n$-th dipoles under the kernel approximation and is given by

$$
\begin{aligned}
& \tilde{Z}_{m, n}=\frac{\jmath \eta}{2 \pi \sin ^{2}(k h)} \int_{-h}^{h} f(z) d z \\
& f(z)=\left(\frac{e^{-\jmath k S_{1}}}{S_{1}}-\cos (k h) \frac{e^{-\jmath k S_{0}}}{S_{0}}\right) \sin (k(h-|z|)), \\
& S_{0}=\sqrt{d_{m, n}^{2}+z^{2}}, S_{1}=\sqrt{d_{m, n}^{2}+(z-h)^{2}}, \\
& d_{m, n}= \begin{cases}\sqrt{\left(x_{m}-x_{n}\right)^{2}+\left(y_{m}-y_{n}\right)^{2}}, & m \neq n, \\
r & m=n,\end{cases} \\
& m, n=0, \ldots, N,
\end{aligned}
$$

\section{B.1.1 End of Wire Condition}

Analogous to Section A, the constants $\left\{\tilde{C}_{m}\right\}_{m=0}^{N-1}$ are obtained by invoking the end of wire condition to (B.1), yielding

$$
\tilde{C}_{m}=-\left(V_{m}-\tilde{I}_{m}(0) \jmath X_{m}\right) \tan (k h) .
$$

\section{B.1.2 Sinusoidal Distribution}

Substituting for $\tilde{C}_{m}$ in (B.1) from (B.7) can be seen to yield the following current distribution on the $n$-th dipole:

$$
\begin{aligned}
& \tilde{I}_{n}(z)= \tilde{I}_{n}(0) \frac{\sin (k(h-|z|))}{\sin (k h)} \\
&=\tilde{I}_{n}(0)(\cos (k|z|)-\cot (k h) \sin (k|z|)), \\
& n=0, \ldots, N-1 .
\end{aligned}
$$

Hence, the approximated Hallen equations in (B.1) can be expressed as

$$
\sum_{n=0}^{N-1} \tilde{Z}_{m, n} \tilde{I}_{n}(0)=\left(V_{m}-\tilde{I}_{m}(0) \jmath X_{m}\right)
$$

$$
m=0, \ldots, N-1 .
$$




\section{B.1.3 Matrix Form}

Using vector notation, (B.9) can be expressed as

$$
\boldsymbol{V}=(\tilde{\boldsymbol{Z}}+\jmath \operatorname{diag}\{\boldsymbol{X}\}) \tilde{\boldsymbol{I}}(0),
$$

where $\boldsymbol{V}$ and $\boldsymbol{X}$ are the excitation voltages and load reactances, respectively, $\tilde{\boldsymbol{I}}(0)=$ $\left.\tilde{\boldsymbol{I}}(z)\right|_{z=0}$ is the feed current vector under the sinusoidal approximation, and $\tilde{\boldsymbol{Z}}$ is the matrix whose $(m, n)$-th element is $\tilde{Z}_{m, n}$ given in (B.2).

To express the current distribution vector under the sinusoidal approximation in a form analogous to the one in (A.18), we write

$$
\tilde{\boldsymbol{I}}=\left[\tilde{\boldsymbol{I}}_{0}^{\top}, \ldots, \tilde{\boldsymbol{I}}_{(N-1)}^{\top}\right]^{\top}, \quad \tilde{\boldsymbol{I}}_{n}=\boldsymbol{s}_{d} \tilde{I}_{n}(0),
$$

where the $(Q+1)$-dimensional vector $\boldsymbol{s}_{d}$ is obtained by evaluating (B.8) at the $(Q+1)$ segments

$$
\boldsymbol{s}_{d}=\boldsymbol{c}_{o}-\cot (k h) \boldsymbol{s}_{i}
$$

The vectors $\boldsymbol{c}_{o}$ and $\boldsymbol{s}_{i}$ are defined in (A.6) in Appendix A.

For the sinusoidal current vector, $\tilde{\boldsymbol{I}}$, we note from (B.10) that the $n$-th feed current can be expressed as

$$
\tilde{I}_{n}(0)=\tilde{\boldsymbol{Y}}_{[n,:]} \boldsymbol{V}
$$

where $\tilde{\boldsymbol{Y}}_{[n,:]}$ is the $n$-th row of the matrix

$$
\begin{aligned}
\tilde{\boldsymbol{Y}} & =(\tilde{\boldsymbol{Z}}+\jmath \operatorname{diag}\{\boldsymbol{X}\})^{-1} \\
& =\tilde{\boldsymbol{Z}}^{-1}\left(\mathbf{I}_{N}+\jmath \operatorname{diag}\{\boldsymbol{X}\} \tilde{\boldsymbol{Z}}^{-1}\right)^{-1} .
\end{aligned}
$$

Substituting from (B.13) and (B.14) in (B.11) yields

$$
\tilde{\boldsymbol{I}}=\tilde{\boldsymbol{M}}\left(\mathbf{I}_{N}+\jmath \operatorname{diag}\{\boldsymbol{X}\} \boldsymbol{U}_{f} \tilde{\boldsymbol{M}}\right)^{-1},
$$


where the (tall) matrix $\tilde{\boldsymbol{M}}$ of dimensions $N(Q+1) \times N$ is given by

$$
\tilde{\boldsymbol{M}}=\left(\mathbf{I}_{N} \otimes \boldsymbol{s}_{d}\right) \tilde{\boldsymbol{Z}}^{-1}
$$




\section{Appendix C}

\section{Power Gain as a Rayleigh Quotient}

Towards expressing the gain as an explicit function of the excitation voltages vector, $\boldsymbol{V}$, we begin by noting that the even symmetry of the current distribution implies that

$$
\begin{aligned}
\sum_{q=-Q}^{Q} I_{n}\left(z_{q}\right) & =I_{n}\left(z_{0}\right)+2 \sum_{q=0}^{Q} I_{n}\left(z_{q}\right) \\
& =\boldsymbol{u}_{s} \boldsymbol{I}_{n},
\end{aligned}
$$

where the dimension of $\boldsymbol{u}_{s}=[1,2, \ldots, 2]$ is $Q+1$.

Writing (2.19) in vector notation yields

$$
\begin{aligned}
G_{a}(\phi \mid \boldsymbol{V}, \boldsymbol{X}) & =\frac{\eta(k \Delta)^{2}}{4 \pi} \frac{\left|\boldsymbol{a}^{\dagger}(\phi) \boldsymbol{U}_{s} \boldsymbol{I}\right|^{2}}{\Re\left\{\boldsymbol{V}^{\dagger} \boldsymbol{U}_{f} \boldsymbol{I}\right\}}, \\
& =\frac{\boldsymbol{V}^{\dagger} \boldsymbol{A}(\phi) \boldsymbol{V}}{\boldsymbol{V}^{\dagger} \boldsymbol{B} \boldsymbol{V}},
\end{aligned}
$$

where (C.4) follows from invoking the (exact) expression of $\boldsymbol{I}$ obtained in (A.18) from the Hallen equations, $\boldsymbol{U}_{f}$ is given in (A.16),

$$
\boldsymbol{U}_{s}=\mathbf{I}_{N} \otimes \boldsymbol{u}_{s},
$$

and the $N \times N$ matrices $\boldsymbol{A}$ and $\boldsymbol{B}$ are given by

$$
\begin{array}{r}
\boldsymbol{A}(\phi)=\boldsymbol{\alpha}(\phi) \boldsymbol{\alpha}^{\dagger}(\phi), \boldsymbol{B}=\Re\left\{\boldsymbol{U}_{f} \boldsymbol{T}\right\}, \\
\boldsymbol{\alpha}(\phi)=\frac{k \Delta}{2} \sqrt{\frac{\eta}{\pi}} \boldsymbol{T}^{\dagger} \boldsymbol{U}_{s}^{\dagger} \boldsymbol{a}(\phi),
\end{array}
$$

where $\boldsymbol{T}$ is given in (2.17). 


\section{Appendix D}

\section{Representation of Excitation Voltage vector in Angular Coordinates and Phase shifts}

Let $\boldsymbol{V}$ be a unit vector representing the excitation voltages. This vector can be expressed in terms of magnitudes and phases as follows:

$$
\boldsymbol{V}=\left[v_{0} e^{-\jmath \psi_{0}}, \ldots, v_{N-1} e^{-\jmath \psi_{N-1}}\right]^{\dagger}
$$

where $v_{n}=\left|V_{n}\right|$ and $\psi_{n}=\arg \left(V_{n}\right), n=0, \ldots,(N-1)$.

Since the vector of magnitudes $\boldsymbol{v}=\left[v_{0}, \ldots, v_{N-1}\right]^{\top}$ is unit norm, i.e., $\|\boldsymbol{v}\|=$ 1 , it can be represented in terms of $(N-1)$ angular coordinates denoted by $\boldsymbol{\theta}=$ $\left[\theta_{0}, \ldots \theta_{N-2}\right]^{\top}$, as follows

$$
\begin{aligned}
v_{0} & =\cos \theta_{0} \\
v_{n} & =\prod_{m=0}^{n-1} \sin \theta_{m} \cos \theta_{n}, \quad n=1, \ldots,(N-2) \\
v_{N-1} & =\prod_{n=0}^{N-2} \sin \theta_{n} .
\end{aligned}
$$

For a given $\boldsymbol{v}$, the angles in $\boldsymbol{\theta}$ can be determined recursively. In particular, to find, the angle $\theta_{n}$ can be obtained from the angle $\theta_{n-1}$ using

$$
\theta_{n}=\arccos \left(v_{n} \tan \theta_{n-1}\right), \quad n=1, \ldots,(N-2) .
$$

From (D.2) and (D.1), the voltage vector $\boldsymbol{V}$ can be expressed in terms of the angular coordinates $\boldsymbol{\theta}$ and the phases $\boldsymbol{\psi}=\left[\psi_{0}, \ldots, \psi_{N-1}\right]^{\top}$, as $\boldsymbol{V}(\boldsymbol{\theta}, \boldsymbol{\psi})$. 


\section{Appendix E}

\section{Gradient of the Power Gain with respect to load reactances}

To implement the steepest ascent algorithm in Section 2.4, in this section, we provide an expression for the gradient of the power gain in (2.20) with respect to the reactances of the passive elements $\boldsymbol{X}_{p}$, that is, we wish to obtain an expression for

$$
\nabla_{\boldsymbol{X}_{p}} G_{a}\left(\phi_{0} \mid \boldsymbol{V}, \boldsymbol{X}\right)=\left[\frac{\partial G_{a}\left(\phi_{0} \mid \boldsymbol{V}, \boldsymbol{X}\right)}{\partial X_{r}}\right]_{r \in \mathcal{N}_{p}} .
$$

To obtain the desired expression we invoke the chain rule, whereby we write

$$
\frac{\partial G_{a}\left(\phi_{0}\right)}{\partial X_{r}}=\frac{\partial G_{a}\left(\phi_{0}\right)}{\partial \boldsymbol{I}} \frac{\partial \boldsymbol{I}}{\partial X_{r}}
$$

where $\frac{\partial G_{a}\left(\phi_{0}\right)}{\partial \boldsymbol{I}} \in \mathbb{C}^{1 \times N(Q+1)}$ and $\frac{\partial \boldsymbol{I}}{\partial X_{r}} \in \mathbb{C}^{N(Q+1) \times 1}$.

The derivative of the power gain with respect to the current vector $\boldsymbol{I}$ can be derived from (C.3) as

$$
\begin{array}{r}
\frac{\partial G_{a}\left(\phi_{0} \mid \boldsymbol{V}, \boldsymbol{X}\right)}{\partial \boldsymbol{I}}=\frac{\eta(k \Delta)^{2}}{4 \pi} \frac{\boldsymbol{I}^{\dagger} \boldsymbol{U}_{s}^{\dagger} \boldsymbol{a}\left(\phi_{0}\right) \boldsymbol{a}^{\dagger}\left(\phi_{0}\right) \boldsymbol{U}_{s}}{\Re\left\{\boldsymbol{V}^{\dagger} \boldsymbol{U}_{f} \boldsymbol{I}\right\}} \\
-\frac{\left|\boldsymbol{a}^{\dagger}\left(\phi_{0}\right) \boldsymbol{U}_{s} \boldsymbol{I}\right|^{2} \boldsymbol{V}^{\dagger} \boldsymbol{U}_{f}}{2\left(\Re\left\{\boldsymbol{V}^{\dagger} \boldsymbol{U}_{f} \boldsymbol{I}\right\}\right)^{2}},
\end{array}
$$

while the derivative of the current vector $\boldsymbol{I}$ with respect to the $r$-th reactance is derived from (A.18) as [18]

$$
\begin{aligned}
\frac{\partial \boldsymbol{I}}{\partial X_{r}} & =\frac{\partial}{\partial X_{r}}\left\{\boldsymbol{M}\left(\mathbf{I}_{N}+\jmath \operatorname{diag}\{\boldsymbol{X}\} \boldsymbol{U}_{f} \boldsymbol{M}\right)^{-1} \boldsymbol{V}\right\} \\
& =\boldsymbol{M} \frac{\partial}{\partial X_{r}}\left\{\left(\mathbf{I}_{N}+\jmath \operatorname{diag}\{\boldsymbol{X}\} \boldsymbol{U}_{f} \boldsymbol{M}\right)^{-1}\right\} \boldsymbol{V} .
\end{aligned}
$$


Let $\boldsymbol{C}=\left(\mathbf{I}_{N}+\operatorname{diag}\{\boldsymbol{X}\} \boldsymbol{U}_{f} \boldsymbol{M}\right)$, thus the term $\frac{\partial}{\partial X_{r}}\left\{\left(\mathbf{I}_{N}+\jmath \operatorname{diag}\{\boldsymbol{X}\} \boldsymbol{U}_{f} \boldsymbol{M}\right)^{-1}\right\}$ is expanded as

$$
\begin{aligned}
\frac{\partial \boldsymbol{C}^{-1}}{\partial X_{r}} & =-\boldsymbol{C}^{-1} \frac{\partial \boldsymbol{C}}{\partial X_{r}} \boldsymbol{C}^{-1}, \\
\frac{\partial \boldsymbol{C}}{\partial X_{r}} & =\jmath\left(\operatorname{diag} \frac{\partial \boldsymbol{X}}{\partial X_{r}}\right) \boldsymbol{U}_{f} \boldsymbol{M} .
\end{aligned}
$$

Finally, the derivative of the load reactance vector $\boldsymbol{X}$ with respect to the $r$-th reactance is

$$
\operatorname{diag} \frac{\partial \boldsymbol{X}}{\partial X_{r}}=\boldsymbol{E}_{r m}
$$

where $\boldsymbol{E}_{r m}$ is the all zero matrix except the $(r m)$-th entry, which is equal to 1. 


\section{Bibliography}

[1] R. Harrington. Reactively controlled directive arrays. IEEE Trans. Antennas Propag., 26(3):390-395, May 1978.

[2] G. K. Papageorgiou, D. Ntaikos, and C. B. Papadias. Efficient beamforming with multi-active multi-passive antenna arrays. In Proc. IEEE Int. Wkshp. Signal Processing Adv. Wireless Commun. (SPAWC), pages 1-5, 2018.

[3] S. Zhang, I. Syrytsin, and G. F. Pedersen. Compact beam-steerable antenna array with two passive parasitic elements for $5 \mathrm{G}$ mobile terminals at $28 \mathrm{GHz}$. IEEE Trans. Antennas Propag., 66(10):5193-5203, July 2018.

[4] H. A. Kayani, Q. Gueuning, N. Goreux, D. Vanhoenacker-Janvier, C. Oestges, and C. Craeye. Reconfigurable cellular base station antenna consisting of parasitic radiators. IEEE Trans. Indust. Electronics, 67(8):7083-7093, August 2020.

[5] Chen Sun, A. Hirata, T. Ohira, and N. C. Karmakar. Fast beamforming of electronically steerable parasitic array radiator antennas: theory and experiment. IEEE Trans. Antennas Propag., 52(7):1819-1832, July 2004.

[6] J. Lota, S. Sun, T. S. Rappaport, and A. Demosthenous. 5G uniform linear arrays with beamforming and spatial multiplexing at 28, 37, 64, and $71 \mathrm{GHz}$ for outdoor urban communication: A two-level approach. IEEE Trans. Veh. Technol., 66(11):9972-9985, August 2017. 
[7] F. W. Vook, A. Ghosh, and T. A. Thomas. MIMO and beamforming solutions for 5G technology. In IEEE MTT-S Int. Microwave Symp. (IMS2014), pages $1-4,2014$

[8] E. Yaacoub, M. Husseini, and H. Ghaziri. An overview of research topics and challenges for 5G massive MIMO antennas. In 2016 IEEE Middle East Conf. Antennas Propag. (MECAP), pages 1-4, 2016.

[9] David V. Thiel and Stephanie Smith. Switched parasitic antennas for cellular communications. Artech House, Inc., USA, 2001.

[10] D. Cheng and F. Tseng. Gain optimization for arbitrary antenna arrays. IEEE Trans. Antennas Propag., 13(6):973-974, May 1965.

[11] B. Schaer, K. Rambabu, J. Bornemann, and R. Vahldieck. Design of reactive parasitic elements in electronic beam steering arrays. IEEE Trans. Antennas Propag., 53(6):1998-2003, June 2005.

[12] T. Tang and M. Gunn. Current distribution on a receiving dipole antenna. IEEE Trans. Antennas Propag., 29(5):817-822, September 1981.

[13] D. F. Kelley. Efficient calculation of radiation patterns for reactively-steered array antennas. In IEEE Antennas Propag. Society Symp., volume 3, pages 2635-2638, 2004.

[14] Sophocles J. Orfanidis. Electromagnetic waves and antennas. Rutgers University, 2003.

[15] D. R. Wilton and N. J. Champagne. Evaluation and integration of the thin wire kernel. IEEE Trans. Antennas Propag., 54(4):1200-1206, April 2006. 
[16] C. Butler. Evaluation of potential integral at singularity of exact kernel in thinwire calculations. IEEE Trans. Antennas Propag., 23(2):293-295, March 1975.

[17] H. Lebret and S. Boyd. Antenna array pattern synthesis via convex optimization. IEEE Trans. Signal Processing, 45(3):526-532, March 1997.

[18] Alexander Graham. Kronecker Products and Matrix Calculus: with Applications. Elis Horwood Ltd., New York, 1981. 


\section{PUBLICATIONS}

\section{Research Publications}

Shady Elkamhawy, Ramy H. Gohary, Ioannis Lambadaris, and Aroosh R. Elahi. On the Optimization of Reactively-Loaded Passive Dipoles for Beam Steering for

Future Wireless Applications. Submitted in IEEE Transactions on Antennas and Propagation, January 2021. 